OPEN ACCESS

Edited by:

Dmitri Rouwet,

Istituto Nazionale di Geofisica e Vulcanologia, sezione di Bologna, Italy

Reviewed by:

Yuri Taran,

National Autonomous University of

Mexico, Mexico

María Martínez Cruz,

Costa Rica Volcanological and

Seismological Observatory

(OVSICORI), Costa Rica

${ }^{*}$ Correspondence:

Muga Yaguchi

myaguchi@mri-jma.go.jp

Specialty section: This article was submitted to

Volcanology,

a section of the journa

Frontiers in Earth Science

Received: 15 July 2021 Accepted: 21 October 2021 Published: 03 December 2021

Citation:

Yaguchi $M$, Ohba $T$ and Terada $A$ (2021) Groundwater Interacting at

Depth With Hot Plastic Magma Triggers Phreatic Eruptions at Yugama

Crater Lake of Kusatsu-Shirane Volcano (Japan).

Front. Earth Sci. 9:741742. doi: 10.3389/feart.2021.741742

\section{Groundwater Interacting at Depth With Hot Plastic Magma Triggers Phreatic Eruptions at Yugama Crater Lake of Kusatsu-Shirane Volcano (Japan)}

\author{
Muga Yaguchi ${ }^{1 *}$, Takeshi Ohba ${ }^{2}$ and Akihiko Terada ${ }^{3}$ \\ ${ }^{1}$ Department of Volcanology Research, Meteorological Research Institute, Japan Meteorological Agency, Tsukuba, Japan, \\ ${ }^{2}$ Department of Chemistry, School of Science, Tokai University, Hiratsuka, Japan, ${ }^{3}$ Volcanic Fluid Research Center, School of \\ Science, Tokyo Institute of Technology, Meguro, Japan
}

Interpreting the triggering mechanisms for phreatic eruptions is a key to improving the hazard assessment of crater lakes. Yugama Crater Lake at Kusatsu-Shirane volcano, Japan, is the site of frequent phreatic eruptions with the recent eruptions in 1982-83, 1989, and 1996, as well as volcanic unrest, including earthquake swarms in 2014 and 2018. To understand the magma-hydrothermal interaction beneath Yugama Crater Lake, we analyzed lake waters from November 2005 to May 2021. From 2005 to 2012, Cl and $\mathrm{SO}_{4}$ concentrations decreased slowly, suggesting the development of a self-sealing zone surrounding the crystallizing magma. We focused on $\mathrm{Ca}, \mathrm{Al}$, and $\mathrm{Si}$ concentrations as representatives of the breach and dissolution of minerals comprising the self-sealing zone and the $\mathrm{Mg} / \mathrm{Cl}$ ratio as an indicator for enhanced interaction between groundwater and hot plastic rock within the self-sealing zone. In 2006-2007, the Ca, Al, Si concentrations and the $\mathrm{Mg} / \mathrm{Cl}$ ratio increased. $\mathrm{No} \mathrm{Cl}$ and $\mathrm{SO}_{4}$ increase during this period suggests the selfsealing zone was leached by deep circulating groundwater rather than by magmatic fluids injection. After the 2014 earthquakes, $\mathrm{Ca}, \mathrm{Al}$, and Si increased again but were associated with a significant $\mathrm{Cl}$ increase and a $\mathrm{pH}$ decrease. We believe that the $\mathrm{HCl}$-rich magmatic fluids breached the self-sealing zone, leading to fluids injection from the crystallizing magma to the Yugama crater. During this period, the $\mathrm{Mg} / \mathrm{Cl}$ ratio did not increase, meaning that magmatic fluids ascending from the breached area of the self-sealing zone inhibited deep intrusion of groundwater into the hot plastic rock region. In 2018, magmatic fluids ascended through the self-sealing zone again with less intensity than in 2014. All eruptions since 1982 have been accompanied by a Mg/Cl ratio increase and a Cl decrease, whereas, when a significant $\mathrm{HCl}$ input occurs, as in 2014 , no eruptions and no $\mathrm{Mg} / \mathrm{Cl}$ ratio increase occurred. This demonstrates that the groundwater-hot plastic rock interaction, rather than the magmatic fluids input, played an essential role in triggering phreatic eruptions; i.e., phreatic eruptions can potentially occur without clear signs of fresh magma intrusions.

Keywords: crater lake, phreatic eruption, hydrothermal system, Kusatsu-Shirane volcano, self-sealing zone, crystallizing magma, $\mathrm{Mg} / \mathrm{Cl}$ ratio, geochemical monitoring 


\section{INTRODUCTION}

Phreatic eruptions do not expel juvenile magma and are typically small in scale. For "wet volcanoes" they can be the dominant type of volcanic eruption that sometimes causes hazardous damage (e.g., Caudron et al., 2015; Yamaoka et al., 2016; Mannen et al., 2019). Although understanding the mechanism and forecast of phreatic eruptions are still challenging, previous studies have shown that various processes in magma-hydrothermal systems, such as rapid pressurization of fluids due to contact between groundwater and hot rocks, increased release of magmatic gases, rapid vaporization of superheated water due to decompression, and sealing by precipitation of hydrothermal minerals, play an important role in triggering this type of eruption (e.g., Hedenquist and Henley, 1985; Barberi et al., 1992; Mastin, 1995; Browne and Lawless, 2001; Stix and de Moor, 2018; Ohba et al., 2019a). An active crater lake connected to a magma-hydrothermal system is a suitable site for studying magma-hydrothermal activity. For example, in Ruapehu (New Zealand), Poás (Costa Rica), and Kelut (Indonesia), chemical analyses of crater lakes were applied as a means of volcanic surveillance (e.g., Giggenbach and Glover, 1975; Rowe et al., 1992; Badrudin, 1994; Bernard and Mazot, 2004; Martínez et al., 2000, 2019; Martínez, 2008; Christenson et al., 2010; Rouwet et al., 2017, 2019).

Yugama Crater Lake at Kusatsu-Shirane volcano on Honshu Island, Japan (Figures 1A-C), is known as a frequent phreatic eruption site (e.g., Ohba et al., 2008a; Terada, 2018) despite there is no clear evidence of recent fresh magma intrusion at shallow depths and actively degassing. Yugama Crater Lake is also an important resource for tourism and economic activities. Interpreting the triggering mechanisms for phreatic eruptions, which occur at such crater lakes as Yugama, is essential to improve how to assess the hazard of phreatic activity at crater
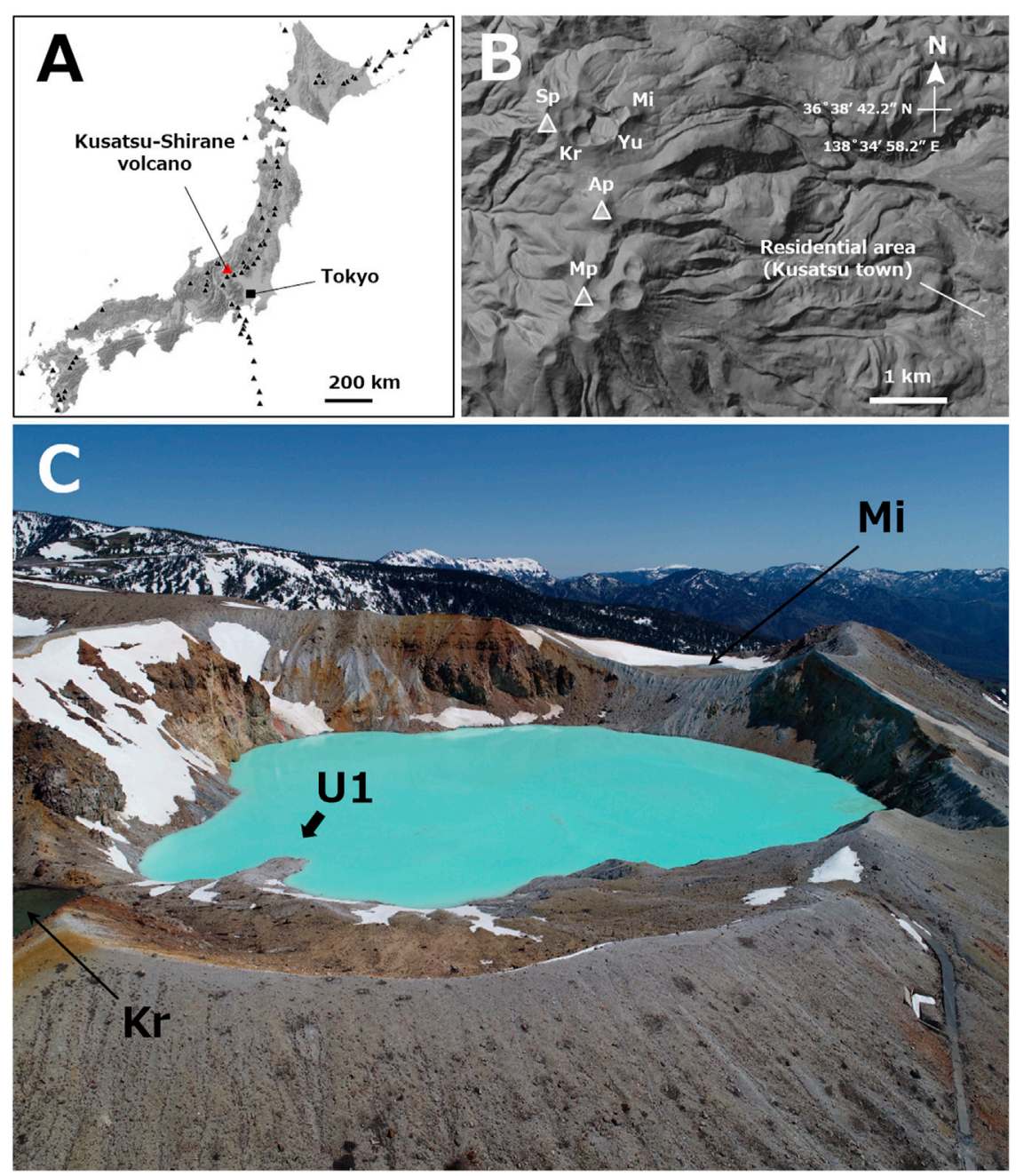

FIGURE 1 | Index map of Kusatsu-Shirane volcano and Yugama crater. (A) Location map of Kusatsu-Shirane volcano (Source of the base map; the Geographical Survey Institute of Japan). Solid triangles indicate an active volcano. (B) Shaded-relief image overlapping the aerial photograph around Kusatsu-Shirane volcano (Source of the base map; the Geographical Survey Institute of Japan). Sp, the peak of Mt. Shirane; Ap, the peak of Mt. Ainomine; Mp, the peak of Mt. Motoshirane; Yu, Yugama crater; Mi, Mizugama crater; and Kr, Karegama. (C) Yugama crater (photograph taken by AT using a drone on May 8, 2020). U1, regular sampling point. 
lake-bearing volcanoes. Accordingly, the correspondence between changes in the chemical composition of lake water and eruptive activity has been investigated at the Yugama crater (e.g., Ohba et al., 1994, 2000, 2008a). For example, in the last 50 years, a phreatic eruption occurred within the Mizugama crater adjacent to the Yugama crater in 1976, and phreatic eruptions occurred within and surrounding the Yugama crater in 1982-83, 1989, and 1996 (Ida et al., 1989; KusatsuShirane Volcano Observatory, Tokyo Institute of Technology, 1996, 1997; Terada, 2018). No eruption has occurred at the Yugama crater since then, but there were earthquake swarms beneath the Yugama crater in 1989-91, followed by a $\mathrm{pH}$ drop in the lake water (Ohba et al., 1994, 2000). For this series of activities, Ohba et al. (2008a) proposed the following model, based on the chemical analysis of lake water at crater Yugama. The 1982-83 eruption was triggered by vapor expansion following the interaction between groundwater and the hot plastic rock region inside the self-sealing zone. The 1989-91 earthquakes and subsequent $\mathrm{pH}$ drops were caused by the groundwater that intruded within the self-sealing zone, extracting $\mathrm{HCl}$-rich fluids from the hot plastic rock region and supplying it to the Yugama crater.

If development and breach of the self-sealing zone play key roles in the magma-hydrothermal activity beneath the Yugama crater, this cycle may continue until the underlying magma cools and loses its volatiles. As expected, earthquake swarms reoccurred around the Yugama crater from March 2014 and in April 2018. Ohba et al. (2019b) estimated that the earthquake swarms in 2014 and 2018 were triggered by the increased pressure in the hydrothermal reservoir due to the injection of magmatic gas, a model based on the analytical results of the chemical and stable isotopic compositions of the fumarolic gases near the Yugama crater. This result made us expect that magma-hydrothermal interaction can be inferred through the chemical composition of Yugama Crater Lake because magmatic gas contains many watersoluble components. In this study, we present the chemical composition of water at Yugama Crater Lake, which was sampled almost every month from November 2005 to May 2021 except for the volcanic unrest period and snow season, and based on this, we investigate the magma-hydrothermal interaction beneath Yugama Crater Lake during this period and propose a key mechanism for the recent eruptions at this crater.

\section{STUDY AREA}

\section{Kusatsu-Shirane Volcano and Hydrothermal Systems Beneath the Yugama Crater}

Kusatsu-Shirane volcano is an active volcano consisting of the pyroclastic cones of Shirane, Ainomine, and Motoshirane (Figure 1B). The eruption stages of this volcano are distinguished into the following: 0.6-0.5 Ma, 0.5-0.3 Ma, and 16 ka-present (Hayakawa and Yui, 1989; Kaneko et al., 1991; Takahashi et al., 2010). Three pyroclastic cones were formed since

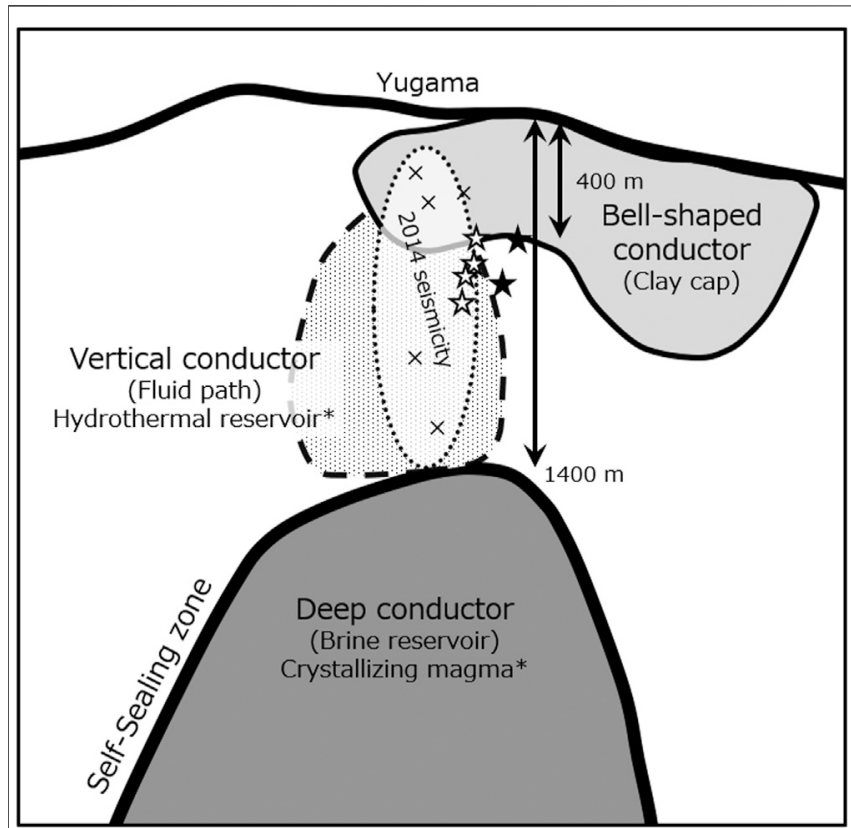

FIGURE 2 | Major structures beneath the Yugama crater inferred by the magnetotellurics survey simplified from Tseng et al. (2020). Cross marks indicate simplified earthquake locations. Open and solid stars indicate approximate locations of magnetizations and demagnetizations corresponding to the volcanic events in 1982-2012 (Takahashi and Fujii, 2014), respectively. ${ }^{*}$ The deep conductor and vertical conductor correspond to crystallizing magma and a hydrothermal reservoir (Ohba et al., 2008a; Ohba et al., 2019b).

$16 \mathrm{ka}$, and recent studies have shown that the Mt. Motoshirane and Mt. Shirane experienced magmatic eruptions at approximately 1.5 and $1.4 \mathrm{ka}$, respectively (Ishizaki et al., 2020; Kametani et al., 2021). The Yugama crater is located near the summit of Mt. Shirane (Figure 1B). All recorded (since 1805) eruptions were phreatic, and almost all occurred in and around the Yugama crater, except for the phreatic eruption at Mt. Motoshirane on January 23, 2018 (e.g., Uto et al., 1983; Japan Meteorological Agency, 2013; Terada, 2018). The last eruption at the Yugama crater was in 1996, but turbulence on the lake surface, such as a dome-shaped water bulge in 1997 and a water jet in 2004, have been observed (Kusatsu-Shirane Volcano Observatory, Tokyo Institute of Technology, 1996, 1997; Volcanic Fluid Research Center, Tokyo Institute of Technology, 2004).

The hydrothermal structure beneath Kusatsu-Shirane volcano has been investigated mainly by magnetotelluric (MT) or audiofrequency magnetotellurics (AMT) methods (Nurhasan et al., 2006; Matsunaga et al., 2020; Tseng et al., 2020). Among these, Tseng et al. (2020) investigated the electrical conductivity structure specifically focused on just beneath the Yugama crater and pointed out three major conductors: a bell-shaped conductor from near the surface to about $400 \mathrm{~m}$ below the surface (m.b.s.), interpreted as the smectite-rich layer; a deep conductor at $>1,500$ m.b.s., interpreted as the brine reservoir; and a vertical conductor connecting the deep conductor and the shallow 

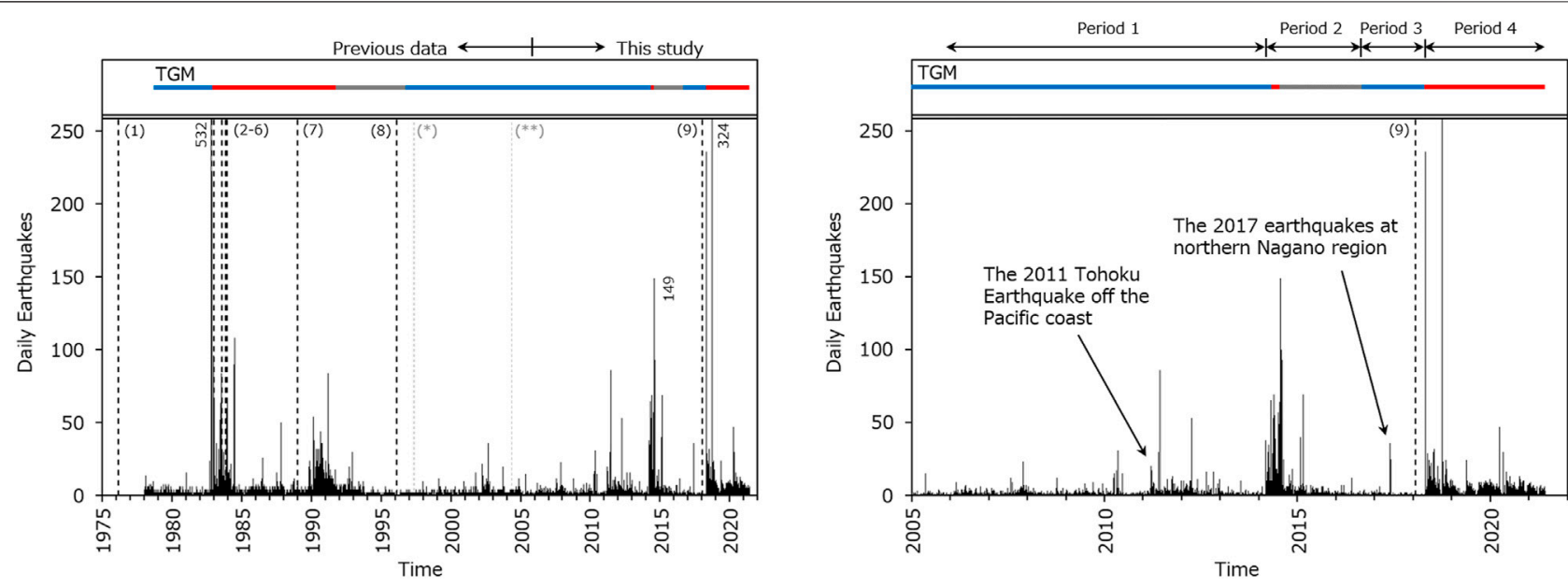

FIGURE 3|Seismicity and thermal activity around the Yugama crater. The number of earthquakes from January 1978 to May 2017 are earthquakes that occurred in the Kusatsu-Shirane volcano, and earthquakes after June 2017 are earthquakes that occurred near the Yugama crater. The 2011 off the Pacific coast of Tohoku Earthquake and the 2017 earthquakes in northern Nagano region are not earthquakes that occurred near the Yugama crater. TGM; Thermal activity beneath the Yugama crater inferred from geomagnetic measurements. Blue periods indicate a magnetizing trend (cooling periods); red periods indicate a demagnetizing trend (heating periods); and gray periods indicate an unclear or stagnant period. Vertical dashed lines indicate eruptive events: (1), the eruption at Mizugama crater in 1976; (2-6), eruptions at the Yugama and the Karegama crater in 1982-1983; (7), a small eruption at the Yugama crater in 1989; (8), a small eruption at the Yugama crater in 1996; and (9), an eruption at Mt. Motoshirane peak in 2018. Vertical thin dashed lines indicate the turbulence on the lake surface: $\left(^{\star}\right)$, water dome in 1997; and (**), water jet in 2004.

conductor (Figure 2). Such hydrothermal structures have also been suggested by geochemical studies of lake water in the Yugama crater and volcanic gases around the Yugama crater (Ohba et al., 2000; Ohba et al., 2008a; Ohba et al., 2019b). Considering this information, a deep conductor corresponds to magma and the surrounding hot plastic rock region, and a vertical conductor corresponds to a two-phase hydrothermal reservoir of liquid and vapor. Flushing of magmatic fluids from deep conductors is thought to be controlled by self-sealing due to mineral deposition.

Volcanic earthquakes have occurred at mainly $1,000 \mathrm{~m}$ beneath the Yugama crater (Japan Meteorological Agency, 2019; Terada et al., 2021). Source locations of thermal magnetization and demagnetization are estimated to be about 400-700 m beneath the Mizugama crater (Takahashi and Fujii, 2014). The seismicity and thermal activity indicate a magmatic fluids injection into the hydrothermal reservoir due to a breach of the self-sealing zone (Ohba et al., 1994, 2008a, 2019b; Tseng et al., 2020).

\section{Overview of Recent Activities of Kusatsu-Shirane Volcano Seismic and Thermal Activity}

As phreatic eruptions have occurred mainly at Mt. Shirane in the last 200 years, geodetic, seismic, and geomagnetic observations have been carried out around the Yugama crater since the 1970s (Ohchi, 1987; Japan Meteorological Agency, 2013). In this study, to discuss the lake water chemistry, we identified the periods of volcanic activity into Periods $1-4$ based on the seismic and the geomagnetic observations (Figure 3). Thermal activity in Figure 3 shows the results of geomagnetic observations simplified into demagnetization (heating), magnetization (cooling), and stagnation.

\section{Period 1 (November 2005-February 2014)}

Although seismic activity, such as after the 2011 Tohoku Earthquake off the Pacific coast (about $420 \mathrm{~km}$ northeast of Kusatsu-Shirane volcano) has been observed, the seismicity from November 2005 to February 2014 was essentially less active (Figure 3). The low seismic activity was consistent with the geomagnetic observations, indicating cooling beneath the Yugama crater during this period (Takahashi and Fujii, 2014; Kakioka Magnetic Observatory, 2016).

\section{Period 2 (March 2014-August 2016)}

Earthquake swarms occurred at Mt. Shirane from March to August 2014, followed by minor seismic activity until March 2018 (the end of Period 3; Figure 3). The thermal demagnetization was observed beneath the Yugama crater from May to June 2014. The thermal demagnetization appeared to stop after July 2014 (Kakioka Magnetic Observatory, 2019).

\section{Period 3 (September 2016-March 2018)}

Minor seismicity at Mt. Shirane was still observed during this period. Although many earthquakes were observed in May 2017 (Figure 3), these earthquakes corresponded to the seismicity at the northern part of Nagano Prefecture, more than $5 \mathrm{~km}$ north of Kusatsu-Shirane volcano (Japan Meteorological Agency, 2018). A magnetized trend was observed from summer in 2016 to March 2018. We consider that the beginning of the magnetization was from September 2016, the same as Period 2 (Kakioka Magnetic Observatory, JMA, 2019). 
On January 23, 2018, a phreatic eruption occurred at Mt. Motoshirane, $2 \mathrm{~km}$ south of the Yugama crater. No precursors, such as changes in seismicity, ground deformations, and thermal demagnetizations, were observed at the Kusatsu-Shirane volcano preceding this eruption (Ogawa et al., 2018; Terada et al., 2021).

\section{Period 4 (April 2018-May 2021)}

Three months after the eruption of Mt. Motoshirane, an earthquake swarm started in late April 2018 at Mt. Shirane (Terada et al., 2021). Seismic activity remained high until May 2021; especially, many earthquakes occurred in May 2019 and March 2020 (Figure 3). A demagnetized trend during this period suggests that a temperature increase in the subsurface of the crater continued from April 2018 to May 2021 (Kakioka Magnetic Observatory, 2019; Japan Meteorological Agency, 2021).

\section{Crustal Deformation}

Crustal deformations have been observed at the Kusatsu-Shirane volcano using tiltmeters and GNSSs. According to tiltmeter observations, the shallow inflation of $1.2 \times 10^{5} \mathrm{~m}^{3}$ beneath the Yugama crater (about 1,000 m.b.s.) was observed from March 2014 to approximately October 2016 (Terada et al., 2021). Such inflation has been detected intermittently also from April 2018, but its inflated volume is one-third of the one of 2014-2016 (Kusatsu-Shirane Volcano Observatory, Tokyo Institute of Technology, 2021).

Preceding the earthquake swarms in 2014 and 2018, the deep pressure source at about $2 \mathrm{~km}$ northwest of the Yugama crater, at a depth of about $6.3 \mathrm{~km}$ below the lake surface, was inflated (Munekane, 2021). The inflated volumes from late-2013 to mid2015 and from late-2017 to mid-2020 are estimated at $5 \times 10^{6} \mathrm{~m}^{3}$ and $2.5 \times 10^{6} \mathrm{~m}^{3}$, respectively. We consider that the deep inflation source affected the earthquake swarms in 2014 and 2018.

\section{MATERIALS AND METHODS}

This study presents the chemical composition of lake water within the Yugama crater from November 2005 to May 2021. The sampling frequency is usually at least once a month, but samples from December to March were usually unavailable because snow inhibits access to the sampling site. The lake water was basically collected using a plastic cup with a long handle on the southwest coast (U1; Figure 1C) of Yugama Crater Lake, but since 2017, remote sampling using a drone, as in Terada et al. (2018), was also applied. The analyses of lake water collected from November 2005 to August 2009 were conducted at Tokyo Institute of Technology, as in Ohba et al. (2008a), and samples collected after this period were analyzed at the Meteorological Research Institute (MRI). At the MRI, the $\mathrm{pH}$ and electrical conductivity were measured using a handheld $\mathrm{pH} / \mathrm{EC}$ meter (Horiba, D-74) equipped with a glass electrode (Horiba, 9615S-10D) calibrated with $\mathrm{pH} 1.68$ and 4.00 buffer solutions and a platinum-platinum black electrode (Horiba, 3552-10D), respectively. The lake waters were filtered with $0.45-\mu \mathrm{m}$ membrane filters and diluted with ultra-pure water using the appropriate dilution factor to bring the concentrations of the analytes to within the calibration ranges and limit of detections (LOD; Table 1), and then subjected to ion chromatography (Thermo Scientific, Dionex Integrion HPIC) to determine $\mathrm{Cl}$ and $\mathrm{SO}_{4}$ concentrations, and microwave plasma atomic emission spectrometer (Agilent, $4210 \mathrm{MP}-\mathrm{AES}$ ) to determine $\mathrm{Na}, \mathrm{K}, \mathrm{Ca}$, $\mathrm{Mg}, \mathrm{Fe}, \mathrm{Al}, \mathrm{Mn}$, and $\mathrm{Si}$ (expressed as $\mathrm{SiO}_{2}$ in Table 2) concentrations. The relative standard deviations (RSD) of IC and MP-AES analyses were better than 3\% (Table 1). Stable isotope ratios $\left(\delta \mathrm{D}\right.$ and $\left.\delta^{18} \mathrm{O}\right)$ of lake waters sampled after 2012 were analyzed by using a cavity ring-down spectrometer (Picarro, L2140-i) and expressed in per mil (\%) relative to VSMOW. Analytical precisions tested were within $\pm 0.4 \%$ o for $\delta \mathrm{D}$ and $\pm 0.05 \%$ o for $\delta^{18} \mathrm{O}$. The analytical results are listed in Table 2.

\section{RESULTS}

\section{Period 1 (November 2005-February 2014)}

The lake water's $\mathrm{pH}$ is approximately $1.2-1.3$, and no significant change was observed (Table 2 and Figure 4). The concentrations of anions, $\mathrm{Cl}$ and $\mathrm{SO}_{4}$, showed a gradual decreasing trend throughout the period with short-term fluctuations (Figure 5A). The concentrations of $\mathrm{Na}, \mathrm{K}, \mathrm{Fe}$, and $\mathrm{Mn}$ did not change significantly, but $\mathrm{Si}$ and certain cations, such as $\mathrm{Al}, \mathrm{Ca}$, and $\mathrm{Mg}$, increased temporarily from September 2006 to November 2007 (Figure 5B).

\section{Period 2 (March 2014-August 2016)}

The $\mathrm{pH}$ decreased sharply (Figure 4), and almost all concentrations of anions, cations, and $\mathrm{SiO}_{2}$ increased (Figure 5). Among these, the decrease in $\mathrm{pH}$ and increase in $\mathrm{Cl}$ concentrations reach values of $\mathrm{pH}=0.91$ and $\mathrm{Cl}=5,180 \mathrm{mg} / \mathrm{L}$ on August 7, 2016, respectively, which is the lowest and highest compared to past observations (Ohba et al., 2008a and references therein), respectively. At the same date, $\delta \mathrm{D}=-39.4 \%$ and $\delta^{18} \mathrm{O}=$ $-1.7 \%$ o (Table 2 ) were observed, which are the highest values ever observed.

\section{Period 3 (September 2016-March 2018)}

The concentrations of dissolved components that increased in Period 2 maintained high values, with fluctuations, until October 2016. Thereafter, the $\mathrm{pH}$ increased until the end of this period, and the concentrations of various constituents decreased in general, with fluctuations (Figures 4, 5).

\section{Period 4 (April 2018-May 2021)}

The overall trend in this period was a persistent decrease in $\mathrm{Cl}$ and $\mathrm{SO}_{4}$ concentrations, which started in August-October 2016. However, after the earthquake swarms (e.g., in April-September 2018, May 2019, and April 2020), an increase in $\mathrm{Cl}$ and $\mathrm{SO}_{4}$ concentration (or stagnation of concentration decrease) occurred. The acceleration in speed of such increases in $\mathrm{Cl}$ and $\mathrm{SO}_{4}$ concentrations after the earthquake swarms were accompanied by increases in $\mathrm{Ca}, \mathrm{Al}, \mathrm{Fe}$, and $\mathrm{Si}$ concentrations (Figure 5). 
TABLE 1 | Analytical performance of anions, cations and Si analyzed at the MRI

\begin{tabular}{|c|c|c|c|c|c|}
\hline Analyte & Technique & $\begin{array}{l}\text { Dilution } \\
\text { factor }\end{array}$ & $\begin{array}{c}\text { Calibration range } \\
\mathrm{mg} / \mathrm{L}\end{array}$ & $\begin{array}{l}\text { LOD } \\
\mathrm{mg} / \mathrm{L}\end{array}$ & $\begin{array}{c}\text { RSD } \\
\%\end{array}$ \\
\hline $\mathrm{Cl}$ & IC & 1,000 and 500 & $0-10$ & (a) $5.05 \times 10^{-3}$ & 0.15 \\
\hline $\mathrm{SO}_{4}$ & IC & 100 and 50 & $0-40$ & (a) $2.04 \times 10^{-2}$ & 0.25 \\
\hline $\mathrm{Al}$ & MP-AES & 40 & $0-10$ & (b) $4.92 \times 10^{-4}$ & 1.54 \\
\hline $\mathrm{Ca}$ & MP-AES & 40 & $0-5$ & (b) $4.42 \times 10^{-4}$ & 0.45 \\
\hline $\mathrm{Fe}$ & MP-AES & 40 & $0-5$ & (b) $8.19 \times 10^{-4}$ & 2.59 \\
\hline K & MP-AES & 40 & $0-1$ & (b) $1.72 \times 10^{-4}$ & 0.87 \\
\hline $\mathrm{Mg}$ & MP-AES & 40 & $0-1$ & (b) $3.97 \times 10^{-4}$ & 0.90 \\
\hline $\mathrm{Mn}$ & MP-AES & 40 & $0-1$ & (b) $5.56 \times 10^{-4}$ & 1.31 \\
\hline $\mathrm{Na}$ & MP-AES & 40 & $0-1$ & (b) $4.35 \times 10^{-4}$ & 0.69 \\
\hline $\mathrm{Si}$ & MP-AES & 40 & $0-5$ & (b) $9.01 \times 10^{-3}$ & 2.32 \\
\hline
\end{tabular}

${ }^{a} \mathrm{LODS}$ of IC analysis were calculated as three times the peak height to baseline noise $\left(\mathrm{S} / \mathrm{N}=3\right.$ ) at the standard's concentration tested $\left(1 \mathrm{mg} / \mathrm{L}\right.$ for $\mathrm{Cl}$ and $4 \mathrm{mg} / \mathrm{L}$ for $\mathrm{SO} \mathrm{H}_{4}$.

${ }^{b} L O D$ s of MP-AES analysis were calculated as $L O D=3 \sigma C /\left(I_{C}-I_{B}\right)$, where $\sigma$ is the standard deviation of blank's intensity, $I_{C}$ is the standard's intensity, $I_{B}$ is the blank's intensity, $C$ is the standard's concentration tested $(0.1 \mathrm{mg} / \mathrm{L}$ for all analytes).

\section{DISCUSSION}

\section{Assumptions for Discussion}

In the following discussion, we assume that the magma-hydrothermal system is affected by crystallization (cooling), rather than melting magma (Figure 6A). The latest magmatic eruption at Mt. Shirane occurred about 1.4 ka ago. Moreover, no magma intrusions into the shallow depth have been detected since the 1990s (Munekane, 2021; Terada et al., 2021). Additionally, although a large amount of heat of over $120 \mathrm{MW}$ is released during the quiescent period (Ueki and Terada, 2012), concentrations of $\mathrm{SO}_{2}$ in fumarolic gas, as a representative of magmatic gas derived from fresh magma at shallow depth, are low (Ohba et al., 2019b). In the assumed system (Figure 6A), magmatic fluids are degassed through the impermeable hot plastic rock surrounding a crystallizing magma, as Giggenbach (1992a) proposed. Secondary mineral deposition as a self-sealing zone occurs at the margin of the hot plastic rock region at about $370-400^{\circ} \mathrm{C}$ (Fournier, 1999). The self-sealing zone corresponds to brittle to plastic transition: dominantly meteoric water circulates at hydrostatic pressure in brittle rock while dominantly magmatic fluids at lithostatic pressure accumulates in hot plastic rock. Magmatic fluids flushes into the overlying brittle region when episodic and temporary breaching of a normally self-sealed zone occurs. Such magma-hydrothermal structure has not yet been demonstrated in the Kusatsu-Shirane volcano, but a very similar structure has been observed in the deep wells drilled in the Kakkonda geothermal field located in northeast Japan. According to the deepest geothermal well (named WD-1a; total depth of $3,729 \mathrm{~m}$ ) data, the quaternary granitic pluton, which is the heat source of this field, was encountered at 2,860 m.b.s., and the brittle-plastic transition was confirmed at 3,100 m.b.s. at about $380^{\circ} \mathrm{C}$, the maximum temperature of $500-510^{\circ} \mathrm{C}$ was recorded at 3,700 m.b.s. (Muraoka et al., 1998). In the brittle rock region of this well, the deeper fluids have a higher salinity (Komatsu et al., 1998). Saline fluids are also recognized below the brittle-plastic transition; for example, hypersaline liquid (about $40 \mathrm{wt} \%$ total chloride species) has been obtained from 3,708 m.b.s. (Kasai et al., 1998). Those Cl- rich fluids were concentrated in the liquid phase during the crystallization process of the crystallizing magma. In the case of Yugama crater lake, $\mathrm{Cl}$ species released to the brittle rock region would be dominated by $\mathrm{HCl}$. This is because, in the assumed hydrothermal system, magmatic fluids are released from the plastic rock region when the self-sealing zone is breached, i.e., under a hydrostatic pressure of about 150 bar (assuming that the depth of plastic rock is $1,500 \mathrm{~m}$; Tseng et al., 2020); at such low pressure, the silicate-water interaction leads to a high $\mathrm{HCl} / \mathrm{NaCl}$ ratio in the aqueous phase (Shinohara and Fujimoto, 1994).

Anionic species (e.g., $\mathrm{Cl}$ and $\mathrm{SO}_{4}$ ) in the most active volcanic lakes mostly originate from the degassing magma, whereas rockforming species (e.g., $\mathrm{Na}, \mathrm{K}, \mathrm{Ca}, \mathrm{Mg}, \mathrm{Al}, \mathrm{Mn}, \mathrm{Fe}$, and $\mathrm{Si}$ ) originate from the leaching from the rock (Ohba et al., 2008a; Rouwet et al., 2014; Varekamp, 2015). However, it is not always necessary that $\mathrm{SO}_{4}$ in the water of Yugama Crater Lake be derived from the unique origin. This is because the sulfur components initially come to the hydrothermal system from the gas phase, but some of them are removed from the aqueous phase as elemental sulfur, and the precipitation and dissolution of secondary minerals such as sulfates and sulfides contribute to the $\mathrm{SO}_{4}$ concentration in lake water. In addition, some of the sulfur species absorbed by the lake water are dissolved in chemical forms other than $\mathrm{SO}_{4}$, such as polythionates (Takano and Watanuki, 1990; Ohba et al., 2008a; Takano et al., 2008).

Phreatic eruptions may form even in the case of magma crystallizing and driving the shallower hydrothermal circulation. In this case, the $\mathrm{Mg} / \mathrm{Cl}$ ratio can be used as an indicator of water-hot plastic rock contact. We assume that $\mathrm{Cl}$ and $\mathrm{Mg}$ originate from the crystallizing magma. In this situation, an increase in $\mathrm{Mg} / \mathrm{Cl}$ is interpreted to correspond to enhanced interaction between water and a hot plastic rock region relative to the stable $\mathrm{HCl}$ degassing state (Giggenbach and Glover, 1975; Giggenbach, 1996; Rouwet et al., 2014). Increases in the $\mathrm{Mg} / \mathrm{Cl}$ ratio were observed before and after all eruptions at Yugama Crater Lake since 1982 (i.e., in 1982-82, 1989, and 1996) and the water bulge appearance in 1997 (Ohba et al., 2008a). 
TABLE 2 | Chemical and isotopic composition of lake water in Yugama crater sampled from November 2005 to May 2021.

\begin{tabular}{|c|c|c|c|c|c|c|c|c|c|c|c|c|c|c|c|}
\hline Sampling date & $\begin{array}{c}\text { Temp. } \\
{ }^{\circ} \mathbf{C}\end{array}$ & $\mathrm{pH}$ & $\begin{array}{l}\text { EC } \\
S / m\end{array}$ & $\begin{array}{c}\mathrm{Cl} \\
\mathrm{mg} / \mathrm{L}\end{array}$ & $\begin{array}{c}\mathrm{SO}_{4} \\
\mathrm{mg} / \mathrm{L}\end{array}$ & $\begin{array}{c}\mathrm{Al} \\
\mathrm{mg} / \mathrm{L}\end{array}$ & $\begin{array}{c}\mathrm{Ca} \\
\mathrm{mg} / \mathrm{L}\end{array}$ & $\begin{array}{c}\mathrm{Fe} \\
\mathrm{mg} / \mathrm{L}\end{array}$ & $\begin{array}{c}\mathrm{K} \\
\mathrm{mg} / \mathrm{L}\end{array}$ & $\begin{array}{c}\mathrm{Mg} \\
\mathrm{mg} / \mathrm{L}\end{array}$ & $\begin{array}{c}\mathrm{Mn} \\
\mathrm{mg} / \mathrm{L}\end{array}$ & $\begin{array}{c}\mathrm{Na} \\
\mathrm{mg} / \mathrm{L}\end{array}$ & $\begin{array}{l}\mathrm{SiO}_{2} \\
\mathrm{mg} / \mathrm{L}\end{array}$ & $\begin{array}{c}\delta^{18} O \\
\% \text { o }\end{array}$ & $\begin{array}{l}\delta D \\
\% \circ\end{array}$ \\
\hline November 4, 2005 & 17.9 & 1.28 & ND & 2,840 & 1740 & 140 & 94.2 & 86.2 & 11.7 & 20.9 & 0.86 & 23.0 & 163 & ND & ND \\
\hline April 20, 2006 & 8.6 & 1.30 & ND & 2,570 & 1,460 & 129 & 85.6 & 70.9 & 11.1 & 20.4 & 0.87 & 20.1 & 139 & ND & ND \\
\hline May 25, 2006 & 18.3 & 1.31 & ND & 2,500 & 1,350 & 128 & 86.2 & 73.6 & 10.1 & 20.2 & 0.85 & 18.9 & 142 & ND & ND \\
\hline June 15, 2006 & 19.1 & 1.30 & ND & 2,430 & 1,360 & 125 & 84.4 & 68.5 & 10.4 & 19.9 & 0.84 & 18.9 & 133 & ND & ND \\
\hline July 20, 2006 & 23.7 & 1.31 & ND & 2,430 & 1,290 & 124 & 83.2 & 67.9 & 9.3 & 19.3 & 0.82 & 17.9 & 130 & ND & ND \\
\hline August 14, 2006 & 26.8 & 1.29 & ND & 2,470 & 1,390 & 128 & 85.9 & 71.6 & 9.3 & 19.9 & 0.83 & 18.5 & 134 & ND & ND \\
\hline September 5, 2006 & 26.4 & 1.29 & ND & 2,490 & 1,390 & 127 & 86.1 & 71.0 & 10.1 & 20.1 & 0.84 & 19.2 & 134 & ND & ND \\
\hline October 13, 2006 & 18.7 & 1.29 & ND & 2,460 & 1,480 & 132 & 89.6 & 72.3 & 10.3 & 23.8 & 1.04 & 20.5 & 137 & ND & ND \\
\hline November 18, 2006 & 16.1 & 1.25 & ND & 2,520 & 1,590 & 135 & 94.0 & 73.6 & 10.0 & 27.9 & 1.25 & 22.1 & 154 & ND & ND \\
\hline April 20, 2007 & 11.2 & 1.27 & ND & 2,640 & 1,580 & 150 & 97.7 & 63.7 & 12.9 & 30.0 & 1.27 & 20.0 & 164 & ND & ND \\
\hline May 31, 2007 & 17.1 & 1.27 & ND & 2,600 & 1,520 & 152 & 100 & 64.4 & 11.4 & 30.0 & 1.25 & 21.3 & 171 & ND & ND \\
\hline June 27, 2007 & 22.8 & 1.27 & ND & 2,590 & 1,500 & 152 & 100 & 65.3 & 15.3 & 30.3 & 1.28 & 22.1 & 169 & ND & ND \\
\hline July 19, 2007 & 25.5 & 1.27 & ND & 2,620 & 1,470 & 158 & 101 & 69.1 & 11.5 & 29.7 & 1.25 & 22.1 & 168 & ND & ND \\
\hline August 21, 2007 & 26.9 & 1.25 & ND & 2,720 & 1,540 & 166 & 103 & 70.0 & 12.0 & 30.7 & 1.32 & 23.5 & 187 & ND & ND \\
\hline September 19, 2007 & 24.9 & 1.28 & ND & 2,540 & 1,510 & 162 & 105 & 74.8 & 11.2 & 29.4 & 1.27 & 22.7 & 185 & ND & ND \\
\hline October 21, 2007 & 20.9 & 1.22 & ND & 2,630 & 1,620 & 166 & 108 & 75.6 & 9.7 & 30.4 & 1.24 & 21.2 & 177 & ND & ND \\
\hline November 14, 2007 & 14.8 & 1.23 & ND & 2,550 & 1,670 & 170 & 107 & 76.0 & 11.3 & 30.3 & 1.24 & 21.8 & 181 & ND & ND \\
\hline April 24, 2008 & 11.2 & 1.31 & ND & 2,440 & 1,330 & 142 & 91.0 & 58.0 & 16.5 & 25.1 & 0.80 & 25.1 & 154 & ND & ND \\
\hline May 13, 2008 & 13.6 & 1.29 & ND & 2,450 & 1,340 & 147 & 94.8 & 59.3 & 11.5 & 25.6 & 0.84 & 23.8 & 153 & ND & ND \\
\hline June 11, 2008 & 20.1 & 1.30 & ND & 2,360 & 1,290 & 138 & 94.6 & 58.8 & 11.5 & 24.5 & 0.79 & 22.6 & 149 & ND & ND \\
\hline July 16, 2008 & 25.2 & 1.35 & ND & 2,340 & 1,330 & 132 & 90.9 & 56.5 & 13.0 & 23.0 & 0.87 & 21.9 & 144 & ND & ND \\
\hline August 15, 2008 & 26.9 & 1.34 & ND & 2,370 & 1,330 & 135 & 93.5 & 61.5 & 13.8 & 23.1 & 0.88 & 22.6 & 148 & ND & ND \\
\hline September 2, 2008 & 25.5 & 1.33 & ND & 2,320 & 1,430 & 146 & 100 & 68.4 & 12.5 & 25.0 & 0.93 & 24.4 & 153 & ND & ND \\
\hline October 21, 2008 & 20.4 & 1.30 & ND & 2,460 & 1,590 & 144 & 97.1 & 72.3 & 10.2 & 25.3 & 0.75 & 22.7 & 153 & ND & ND \\
\hline November 7, 2008 & 15.3 & 1.28 & ND & 2,520 & 1,630 & 147 & 96.3 & 72.2 & 6.5 & 25.3 & 0.75 & 23.2 & 159 & ND & ND \\
\hline April 23, 2009 & 12.1 & 1.30 & ND & 2,540 & 1,410 & 136 & 89.9 & 57.7 & 11.2 & 23.4 & 0.78 & 22.3 & 142 & ND & ND \\
\hline May 21, 2009 & 19.0 & 1.30 & ND & 2,560 & 1,360 & 140 & 92.0 & 59.6 & 11.1 & 23.7 & 0.86 & 23.0 & 149 & ND & ND \\
\hline June 9, 2009 & 20.2 & 1.29 & ND & 2,570 & 1,290 & 140 & 92.8 & 59.0 & 10.2 & 23.8 & 0.94 & 23.4 & 149 & ND & ND \\
\hline July 16, 2009 & 22.4 & 1.28 & ND & 2,580 & 1,300 & 144 & 92.3 & 59.8 & 12.1 & 24.5 & 0.92 & 24.0 & 150 & ND & ND \\
\hline August 13, 2009 & 27.1 & 1.29 & ND & 2,570 & 1,300 & 144 & 94.5 & 61.3 & 11.2 & 24.7 & 0.84 & 24.0 & 151 & ND & $N D$ \\
\hline June 26, 2012 & 21.6 & 1.29 & 2.35 & 2,230 & 1,120 & 132 & 92.0 & 64.3 & 10.2 & 19.5 & 0.66 & 22.3 & 161 & -6.4 & -60.2 \\
\hline May 19, 2014 & 18.8 & 1.24 & 2.76 & 2,630 & 1,100 & 124 & 85.2 & 68.0 & 10.4 & 19.9 & 0.70 & 21.1 & 162 & -5.6 & -58.7 \\
\hline August 23, 2014 & ND & 1.19 & 2.93 & 2,680 & 1,250 & 130 & 92.8 & 75.7 & 10.3 & 20.8 & 0.74 & 22.7 & 168 & -5.0 & -55.1 \\
\hline September 27, 2014 & 25.3 & 1.15 & 3.24 & 3,000 & 1,370 & 144 & 98.9 & 77.7 & 10.3 & 22.8 & 0.77 & 23.3 & 181 & -4.2 & -51.6 \\
\hline May 15, 2015 & 22.6 & 1.07 & 3.97 & 3,630 & 1,310 & 160 & 96.9 & 67.1 & 12.0 & 25.2 & 0.81 & 25.7 & 194 & -4.1 & -48.2 \\
\hline August 28, 2015 & ND & 1.02 & 4.49 & 4,040 & 1,670 & 179 & 105 & 82.2 & 13.2 & 28.0 & 0.92 & 29.4 & 208 & -3.0 & -44.1 \\
\hline October 15, 2015 & 21.4 & 1.01 & 4.62 & 4,170 & 1780 & 178 & 111 & 79.9 & 12.6 & 29.1 & 0.96 & 29.2 & 211 & -2.7 & -42.2 \\
\hline April 22, 2016 & 16.6 & 0.94 & 5.48 & 4,670 & 1890 & 210 & 120 & 79.9 & 14.8 & 33.9 & 1.11 & 33.0 & 219 & -2.1 & -39.5 \\
\hline August 7, 2016 & 29.8 & 0.91 & 5.79 & 5,180 & 2,220 & 217 & 129 & 88.7 & 18.0 & 35.4 & 1.18 & 38.3 & 213 & -1.7 & -39.4 \\
\hline August 25, 2016 & 27.5 & 0.91 & 5.76 & 5,180 & 2,230 & 226 & 132 & 94.1 & 17.0 & 36.5 & 1.21 & 37.0 & 214 & -1.8 & -39.5 \\
\hline September 6, 2016 & 26.5 & 0.91 & 5.71 & 5,000 & 2,200 & 222 & 133 & 94.3 & 16.8 & 36.1 & 1.19 & 36.2 & 217 & -1.8 & -39.5 \\
\hline September 29, 2016 & 24.9 & 0.94 & 5.42 & 4,900 & 2,140 & 219 & 131 & 95.0 & 17.1 & 34.4 & 1.22 & 37.4 & 211 & -2.5 & -42.6 \\
\hline October 17, 2016 & 21.9 & 0.92 & 5.55 & 5,080 & 2,230 & 226 & 132 & 104 & 17.0 & 36.9 & 1.17 & 37.0 & 215 & -2.1 & -41.3 \\
\hline October 27, 2016 & 17.4 & 0.92 & 5.69 & 5,140 & 2,230 & 236 & 137 & 102 & 20.0 & 37.5 & 1.26 & 45.7 & 221 & -1.8 & -40.0 \\
\hline April 25, 2017 & 13.8 & 0.96 & 4.97 & 4,370 & 1790 & 205 & 121 & 81.3 & 14.1 & 32.2 & 1.08 & 31.7 & 170 & -3.9 & -48.5 \\
\hline May 17, 2017 & 17.0 & 0.98 & 5.14 & 4,750 & 1840 & 200 & 122 & 81.0 & 13.7 & 31.8 & 1.09 & 33.5 & 176 & -3.6 & -47.3 \\
\hline July 21, 2017 & 26.5 & 0.97 & 5.02 & 4,460 & 1810 & 203 & 121 & 82.3 & 14.2 & 32.3 & 1.08 & 33.8 & 179 & -3.6 & -48.3 \\
\hline September 13, 2017 & 24.0 & 1.00 & 4.76 & 4,250 & 1780 & 199 & 118 & 82.2 & 13.5 & 30.3 & 1.03 & 30.9 & 175 & -3.3 & -47.0 \\
\hline October 5, 2017 & 19.3 & 0.98 & 4.79 & 4,300 & 1800 & 204 & 119 & 89.0 & 13.6 & 31.0 & 1.01 & 32.3 & 177 & -3.2 & -46.4 \\
\hline (a) October 18, 2017 & ND & 1.01 & 4.98 & 4,450 & 1830 & 205 & 120 & 93.4 & 16.7 & 28.4 & 1.31 & 37.5 & 160 & -3.6 & -48.5 \\
\hline November 10, 2017 & 11.7 & 1.00 & 4.75 & 4,200 & 1800 & 194 & 121 & 87.1 & 13.9 & 30.5 & 1.03 & 31.3 & 175 & -3.7 & -49.0 \\
\hline May 18, 2018 & 18.6 & 1.02 & 4.51 & 4,220 & 1,490 & 189 & 120 & 82.4 & 14.8 & 27.9 & 1.17 & 31.4 & 152 & -4.3 & -51.6 \\
\hline June 19, 2018 & 22.5 & 1.01 & 4.54 & 4,340 & 1,550 & 198 & 129 & 86.3 & 14.6 & 29.2 & 1.17 & 32.2 & 158 & -4.0 & -50.7 \\
\hline July 9, 2018 & 24.5 & 1.03 & 4.42 & 4,420 & 1,610 & 226 & 123 & 92.0 & 15.3 & 29.7 & 1.22 & 35.3 & 164 & -4.1 & -51.6 \\
\hline August 2, 2018 & 26.8 & 1.04 & 4.50 & 4,360 & 1,640 & 214 & 134 & 90.9 & 15.9 & 30.4 & 1.15 & 34.3 & 181 & -3.8 & -50.6 \\
\hline October 17, 2018 & 21.4 & 1.03 & 4.35 & 4,270 & 1,620 & 201 & 122 & 93.2 & 13.6 & 30.1 & 0.91 & 29.7 & 189 & -3.6 & -49.4 \\
\hline May 24, 2019 & 18.8 & 1.10 & 4.29 & 4,270 & 1,420 & 211 & 128 & 84.9 & 14.6 & 31.4 & 1.01 & 35.0 & 188 & -3.6 & -47.0 \\
\hline [b] July 1, 2019 & ND & 1.05 & 4.20 & 4,130 & 1,420 & 204 & 139 & 89.5 & 14.5 & 33.4 & 0.98 & 31.3 & 201 & -3.7 & -47.9 \\
\hline [c] July 1, 2019 & ND & 1.05 & 4.21 & 4,100 & 1,410 & 196 & 135 & 85.9 & 16.0 & 32.3 & 0.94 & 44.0 & 195 & -3.7 & -47.9 \\
\hline [d] July 1, 2019 & ND & 1.04 & 4.20 & 4,110 & 1,410 & 194 & 131 & 84.5 & 14.2 & 32.0 & 0.96 & 33.2 & 193 & -3.4 & -47.3 \\
\hline August 2, 2019 & 27.1 & 1.07 & 4.10 & 4,070 & 1,420 & 219 & 148 & 104 & 15.9 & 31.7 & 1.06 & 33.5 & 203 & -3.8 & -49.1 \\
\hline August 26, 2019 & 26.0 & 1.06 & 4.21 & 4,090 & 1,460 & 219 & 144 & 99.4 & 16.7 & 31.8 & 1.09 & 33.2 & 199 & -3.6 & -48.2 \\
\hline October 1, 2019 & 25.0 & 1.06 & 4.24 & 4,160 & 1,550 & 231 & 165 & 118 & 20.6 & 33.4 & 1.25 & 38.0 & 222 & -3.0 & -46.1 \\
\hline
\end{tabular}


TABLE 2 | (Continued) Chemical and isotopic composition of lake water in Yugama crater sampled from November 2005 to May 2021.

\begin{tabular}{|c|c|c|c|c|c|c|c|c|c|c|c|c|c|c|c|}
\hline Sampling date & $\begin{array}{c}\text { Temp. } \\
{ }^{\circ} \mathbf{C}\end{array}$ & $\mathrm{pH}$ & $\begin{array}{c}\text { EC } \\
\text { S/m }\end{array}$ & $\begin{array}{c}\mathrm{Cl} \\
\mathrm{mg} / \mathrm{L}\end{array}$ & $\begin{array}{c}\mathrm{SO}_{4} \\
\mathrm{mg} / \mathrm{L}\end{array}$ & $\begin{array}{c}\mathrm{Al} \\
\mathrm{mg} / \mathrm{L}\end{array}$ & $\begin{array}{c}\mathrm{Ca} \\
\mathrm{mg} / \mathrm{L}\end{array}$ & $\begin{array}{c}\mathrm{Fe} \\
\mathrm{mg} / \mathrm{L}\end{array}$ & $\begin{array}{c}\mathrm{K} \\
\mathrm{mg} / \mathrm{L}\end{array}$ & $\begin{array}{c}\mathrm{Mg} \\
\mathrm{mg} / \mathrm{L}\end{array}$ & $\begin{array}{c}\mathrm{Mn} \\
\mathrm{mg} / \mathrm{L}\end{array}$ & $\begin{array}{c}\mathrm{Na} \\
\mathrm{mg} / \mathrm{L}\end{array}$ & $\begin{array}{c}\mathrm{SiO}_{2} \\
\mathrm{mg} / \mathrm{L}\end{array}$ & $\begin{array}{c}\delta^{18} O \\
\% \text { o }\end{array}$ & $\begin{array}{l}\delta D \\
\% \circ\end{array}$ \\
\hline October 16, 2019 & ND & 1.11 & 3.86 & 3,800 & 1,460 & 230 & 164 & 120 & 17.7 & 30.8 & 1.23 & 35.9 & 196 & -3.9 & -49.7 \\
\hline November 9, 2019 & 15.4 & 1.07 & 3.90 & 3,850 & 1,470 & 234 & 171 & 129 & 17.9 & 32.7 & 1.30 & 38.8 & 204 & -3.7 & -49.4 \\
\hline December 3, 2019 & ND & 1.09 & 4.03 & 3,920 & 1,500 & 230 & 174 & 130 & 17.1 & 31.7 & 1.24 & 35.4 & 202 & -3.3 & -47.8 \\
\hline April 3, 2020 & 8.3 & 1.13 & 3.77 & 3,790 & 1,310 & 206 & 159 & 119 & 15.5 & 32.2 & 1.13 & 34.1 & 193 & -3.8 & -49.8 \\
\hline April 30, 2020 & 14.3 & 1.16 & 3.41 & 3,170 & 1,100 & 180 & 140 & 102 & 15.5 & 28.0 & 0.94 & 30.4 & 169 & -4.6 & -51.8 \\
\hline (b) May 8, 2020 & ND & 1.13 & 3.67 & 3,900 & 1,330 & 222 & 165 & 122 & 16.9 & 31.3 & 1.27 & 36.6 & 184 & -3.9 & -49.5 \\
\hline (c) May 8, 2020 & ND & 1.13 & 3.67 & 3,880 & 1,330 & 221 & 163 & 120 & 16.7 & 31.1 & 1.23 & 36.3 & 184 & -3.5 & -49.3 \\
\hline June 2, 2020 & 20.8 & 1.11 & 3.70 & 3,830 & 1,320 & 225 & 170 & 127 & 16.7 & 32.4 & 1.26 & 35.9 & 189 & -3.7 & -49.9 \\
\hline August 11, 2020 & 26.9 & 1.13 & 3.63 & 3,820 & 1,350 & 221 & 166 & 129 & 18.7 & 31.0 & 1.29 & 37.5 & 190 & -3.6 & -52.1 \\
\hline September 8, 2020 & 27.2 & 1.12 & 3.79 & 3,910 & 1,390 & 235 & 173 & 139 & 17.2 & 33.2 & 1.27 & 38.5 & 196 & -3.3 & -50.0 \\
\hline October 15, 2020 & 21.5 & 1.12 & 3.79 & 3,990 & 1,420 & 243 & 176 & 141 & 17.7 & 33.9 & 1.31 & 39.1 & 200 & -3.3 & -49.4 \\
\hline November 5, 2020 & 16.6 & 1.13 & 3.90 & 4,040 & 1,420 & 238 & 174 & 142 & 17.6 & 33.9 & 1.32 & 39.2 & 199 & -2.8 & -48.0 \\
\hline (b)November 14, 2020 & ND & 1.08 & 3.89 & 4,110 & 1,430 & 240 & 176 & 142 & 17.5 & 34.2 & 1.33 & 38.8 & 206 & -2.9 & -47.3 \\
\hline April 20, 2021 & 13.1 & 1.11 & 3.90 & 4,080 & 1,270 & 205 & 149 & 104 & 15.0 & 32.8 & 1.06 & 32.7 & 197 & -3.6 & -48.5 \\
\hline April 26, 2021 & 13.5 & 1.11 & 3.85 & 4,140 & 1,280 & 242 & 169 & 127 & 17.2 & 34.0 & 1.25 & 38.9 & 198 & -3.5 & -48.8 \\
\hline May 31, 2021 & 20.3 & 1.12 & 3.92 & 4,070 & 1,260 & 227 & 172 & 126 & 17.9 & 33.9 & 1.28 & 40.2 & 203 & -3.4 & -47.4 \\
\hline
\end{tabular}

${ }^{a}$ Data cited from Terada et al. (2018).

${ }^{b}$ Water sampled approximately $10 \mathrm{~m}$ offshore from the U1 point by using a drone.

${ }^{c}$ Water sampled near the center of the crater by using a drone.

${ }^{d}$ Water sampled from the discolored area of the crater by using a drone.

\section{Period 1 (November 2005-February 2014) Development of the Self-Sealing Zone and its Breach due to Leaching by Deep Circulating Water}

During this period, $\mathrm{Cl}$ and $\mathrm{SO}_{4}$ concentrations decreased gradually. Considering the minor seismicity and the cooling of the hydrothermal reservoir inferred by the geomagnetic observation (Takahashi and Fujii, 2014), the supply of magmatic fluids was suppressed due to the development of the self-sealing zone. The gradual decrease in $\mathrm{Cl}$ and $\mathrm{SO}_{4}$ is considered due to dominantly meteoric groundwater circulating in the hydrothermal system only mixing with the residual saline fluids in the deep part of the brittle rock region, and being washed away to the Yugama Crater Lake, with no additional magmatic fluids ascending, as shown in Figure 6A. Considering that the cooling of the hydrothermal reservoir in Period 1 has continued since 1996 (Figure 3; Kakioka Magnetic Observatory, 2016), self-sealing may have started before this study period.

Nevertheless, the $\mathrm{Mg}$ concentration increased from 2006 to 2007 (Figure 5B), resulting in an increase in the $\mathrm{Mg} / \mathrm{Cl}$ ratio (circle "a" in Figure 7). At this time, the increase of $\mathrm{Al}, \mathrm{Ca}$, and $\mathrm{Si}$ was also observed (Figure 5B). As $\mathrm{Al}, \mathrm{Ca}$, and $\mathrm{Si}$ are major components of secondary mineral depositions that constitute the self-sealing zone (e.g., alunite, gypsum/anhydrite or $\mathrm{SiO}_{2}$ ) and $\mathrm{Mg}$ originates dissolution of crystallizing magma, we believe that two processes occurred in 2006: 1) the breach (dissolution) of the selfsealing zone, and 2) the contact between groundwater and the hot plastic rock region inside the self-sealing zone. However, since the increase in $\mathrm{Al}, \mathrm{Ca}$, and $\mathrm{Si}$ concentrations were smaller than before and after the 1982-83 eruptive period (Figure 5B), the breach of the self-sealing zone in 2006 would have been smaller in scale. Moreover, the region below the brittle-plastic transition has a temperature of about $>400^{\circ} \mathrm{C}$ with low permeability, by referring to the deep well in the Kakkonda geothermal field (Komatsu et al., 1998; Muraoka et al., 1998). The saturated vapor temperature of water at $150 \mathrm{bar}$ is about $340^{\circ} \mathrm{C}$; thus, the groundwater that reached the hot plastic rock from the small breached part of the self-sealing zone in 2006 could not invade deeply and abundantly as, otherwise, it would evaporate.

Direct evidence for the presence and mineral species of the self-sealing zone beneath the Yugama Crater Lake has not been revealed by direct drilling, however, ejecta from past phreatic eruptions provide important insights. The abundant phases in eruptive ejecta of Ruapehu Crater Lake (New Zealand), which has repeated phreatic eruptions, are low-temperature $\mathrm{SiO}_{2}$ polymorphs such as cristobalite, sulfates such as gypsum/ anhydrite and alunite, pyrite, and native sulfur (Christenson and Wood, 1993). Especially, native sulfur, alunite, anhydrite, and $\mathrm{SiO}_{2}$ are considered to be readily precipitated in hydrothermal systems (Christenson et al., 2010). Alunite, gypsum/anhydrite, and $\mathrm{SiO}_{2}$ polymorphs are also abundant in the ejecta of the 1982-83 eruptions at the Yugama Crater Lake (Ossaka et al., 1984; Table 3), supporting the presence of a self-sealing zone and its dissolution to enhance the increase of $\mathrm{Al}, \mathrm{Ca}$, and $\mathrm{Si}$ concentrations in Yugama Crater Lake. Considering no increase in $\mathrm{Cl}$ or $\mathrm{SO}_{4}$ before or after the increase of the $\mathrm{Mg} / \mathrm{Cl}$ ratio in 2006 , it is unlikely that the injection of magmatic fluids caused the breach of the selfsealing zone at that time. Therefore, we believe that a long period of suppression of magmatic fluids, arguably also due to the presence of the self-sealing zone, allows groundwater to circulate deeply and allows groundwater to leach the mineral deposition of the self-sealing zone and intrusion into the hot plastic rock region (path "i" in Figure $\mathbf{6 B}_{\mathrm{I}}$ ). Afterward, the self- 

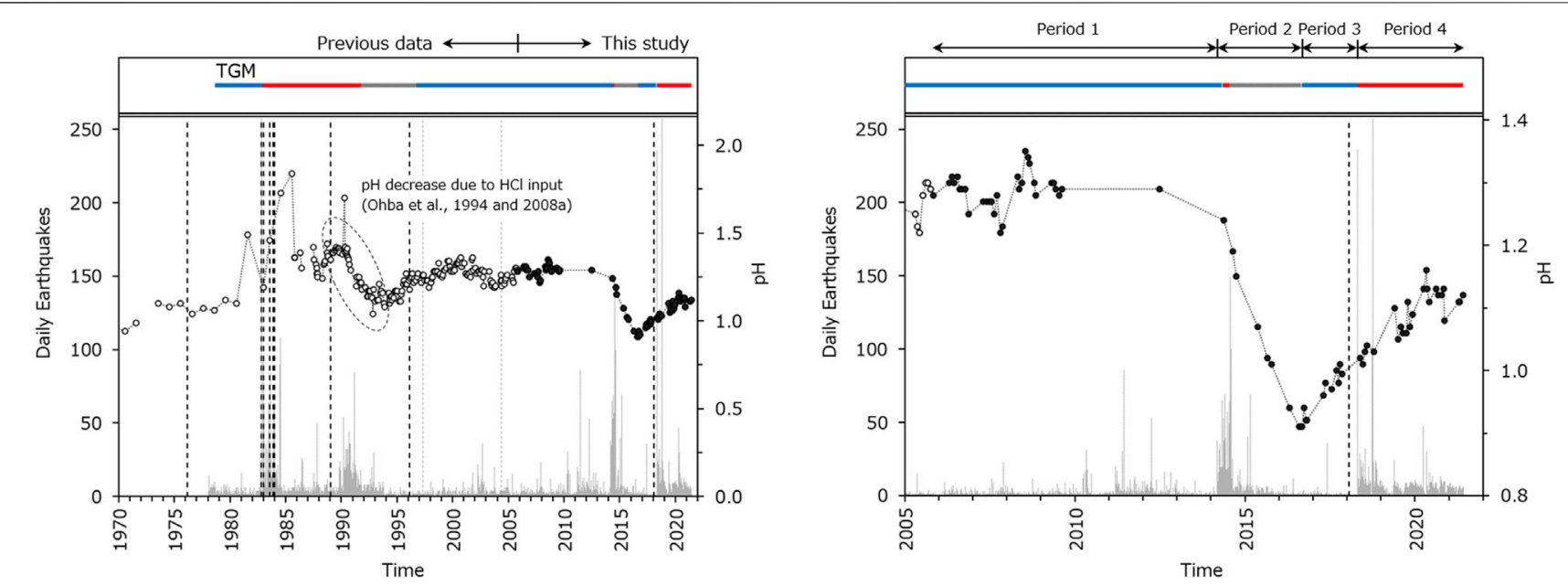

FIGURE 4 | Lake water pH values in Yugama crater. Open and solid circles indicate the previous data since the 1970s (Ohba et al., 2008a) and the present analyses, respectively. TGM and the vertical dashed line show the same as Figure $\mathbf{3}$.
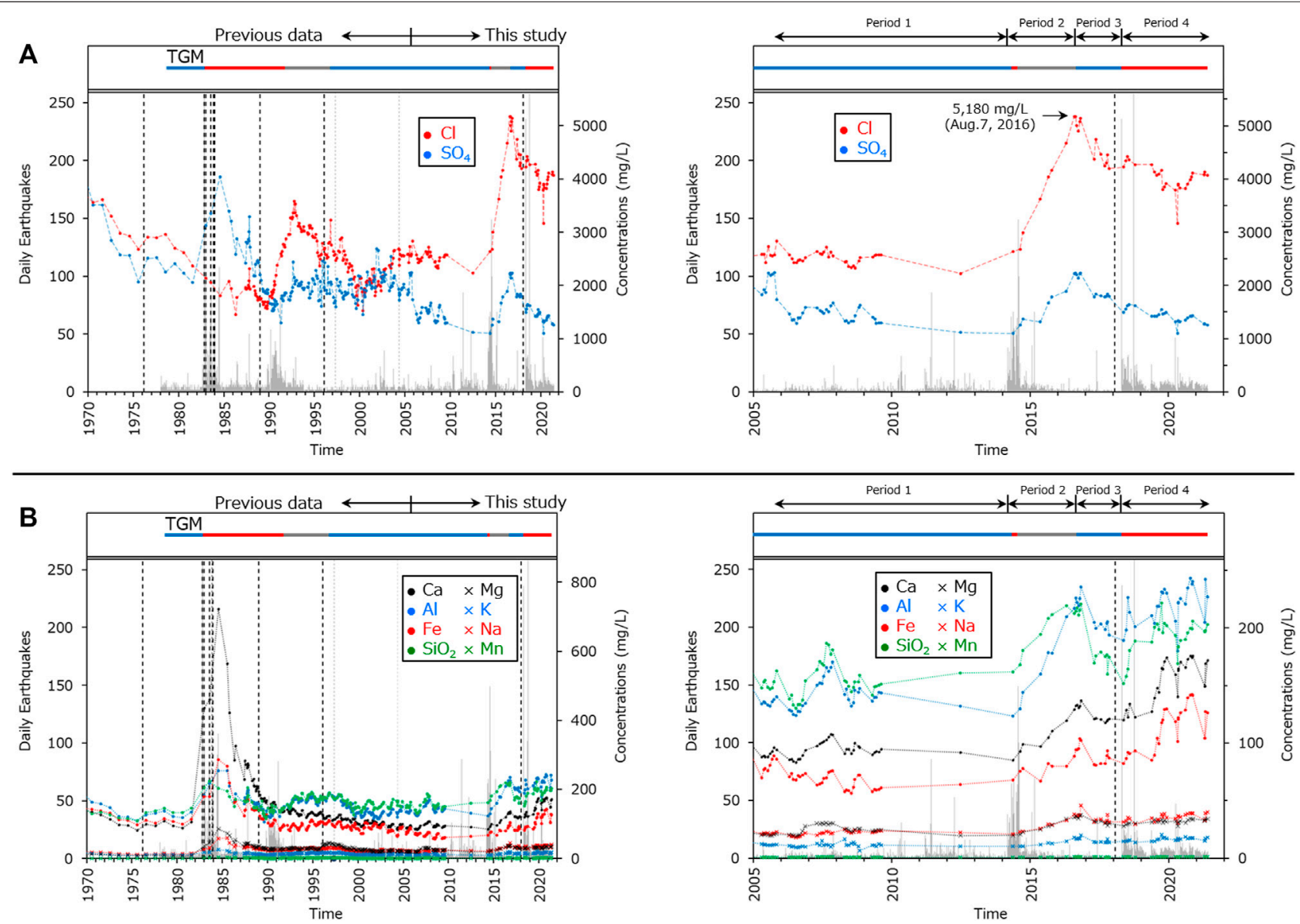

FIGURE 5 | Concentrations of anions (A), cations, and silica (B) of lake water in Yugama crater. Data before October 2005 were cited from Ohba et al. (2008a). TGM and the vertical dashed line show the same as Figure $\mathbf{3}$. 

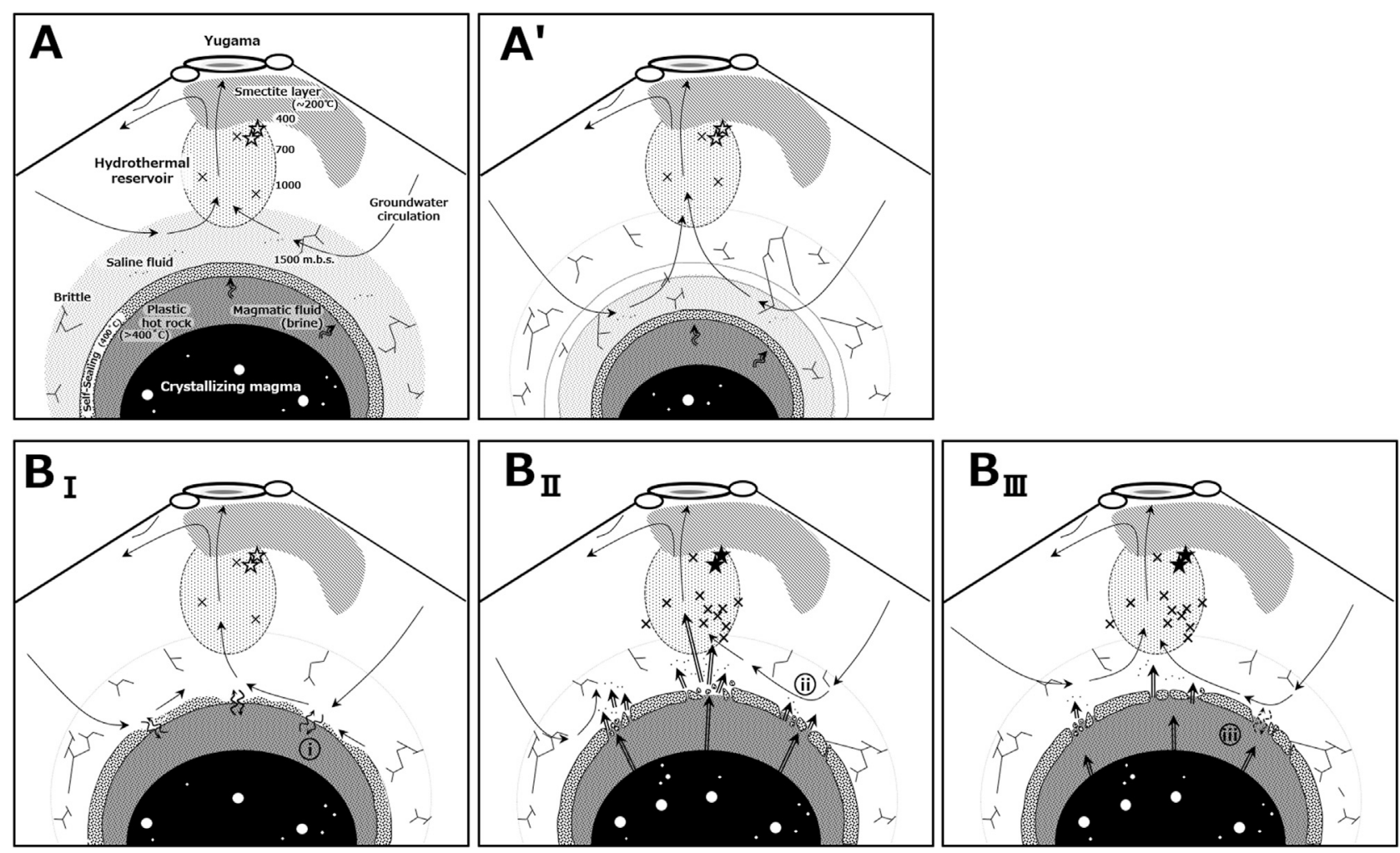

FIGURE 6 | Schematic image for the state of magma-hydrothermal system beneath Yugama Crater Lake modeled after (Giggenbach, 1992a; Fournier, 1999; Ohba et al., 2008a, 2019b). Cross marks indicate simplified earthquake locations. Open and solid stars indicate approximate locations of magnetizations and demagnetizations corresponding to the volcanic events in 1982-2012 (Takahashi and Fujii, 2014), respectively. The cooling magma is surrounded by a hot plastic rock region. A self-sealing zone composed of secondary minerals, such as silica, gypsum/anhydrite, and alunite, separate the hot plastic rock region and the brittle rock region. This boundary plays a role in the advancing front of brittle fracturing. Cl-rich saline fluid expelled from the crystallizing magma remains in the deep part of the brittle region. (A) Development of the self-sealing zone suppresses the ascent of magmatic fluid to the hydrothermal reservoir, resulting in the cooling of the reservoir. The groundwater circulation dilutes the remaining $\mathrm{Cl}$ in the deep in the brittle region and supplies them to the Yugama crater through the hydrothermal reservoir. (B) Breaching phase of the self-sealing zone. $\left(\mathbf{B}_{\mathbf{1}}\right)$ : The groundwater circulation continues to transport $\mathrm{Cl}$ with heat from deep to the Yugama crater and deepens the

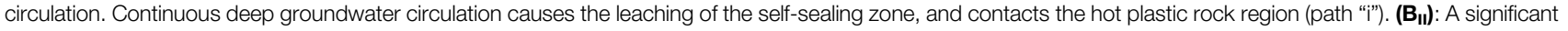
ascent of magmatic $\mathrm{HCl}$-rich fluid breaches the self-sealing zone. Contact between the groundwater and the hot plastic rock region is not enhanced because the deep circulating groundwater is inhibited by the ascending flow of magmatic fluid (path "ii"). ( $\mathbf{B}_{\mathrm{III}}$ ): A less intense ascent of magmatic fluid allows groundwater to intrude slightly into the hot plastic rock region (path "iii"). ( $\mathbf{A}^{\prime}$ ) The self-sealing zone regrows, but its location advances toward the depth through repeated breaches and regrowth. Deeper circulation is needed for the contact between groundwater and hot plastic rock.

sealing zone regrew and returned to the state shown in Figure 6A, until the end of this period.

Although no eruption occurred at the Yugama Crater Lake in 2006 , the above mentioned invasion of groundwater into the brittle magma carapace that envelops the hot plastic rock and the self-sealing zone can trigger hydrothermal destabilization and phreatic eruptions. For example, at the hyperacid crater lake of Poás volcano in Costa Rica, the lake level dropped by about $8 \mathrm{~m}$ accompanied by an increase in seismic activity through 1998, followed by, between late 1998 and mid-1999, the opening of cracks in the ground and new fumaroles, and enhanced influx of heat and volatiles into the lake as indicated by the increase in lake water temperature and concentrations of solutes (Martínez, 2008). At Yugama Crater Lake, the water level was high during the eruption and decreased after the eruption in the active period of 1982-83. In particular, after the October 26, 1982 eruption, the first eruption of the 1982-83 series, the water level decreased remarkably, dropping about $7 \mathrm{~m}$, followed by the subsequent eruption on December 29 (Tokyo Institute of Technology and Sophia University, 1983). The high lake level during the eruption is attributed to the discharge pressure of the volcanic fluids, while the decrease after the eruption is attributed to the deep invasion of lake water, mainly through the conduit created by the eruption (Tokyo Institute of Technology and Sophia University, 1983; Ossaka et al., 1984). The lake level rise during the eruption would be similar to that occurred about a year before the first phreatic eruption at Poás in March 2006, which is interpreted as the pushing vapor front (Rouwet et al., 2017). For the period without eruptions, i.e., when there is no open conduit to a deep hydrothermal system, the depth of groundwater invasion or percolation at Kusatsu-Shirane volcano has not yet been evaluated by geochemical or geophysical observations. On the other hand, other volcanoes and geothermal fields in Japan, such as Mt. Fuji and the 

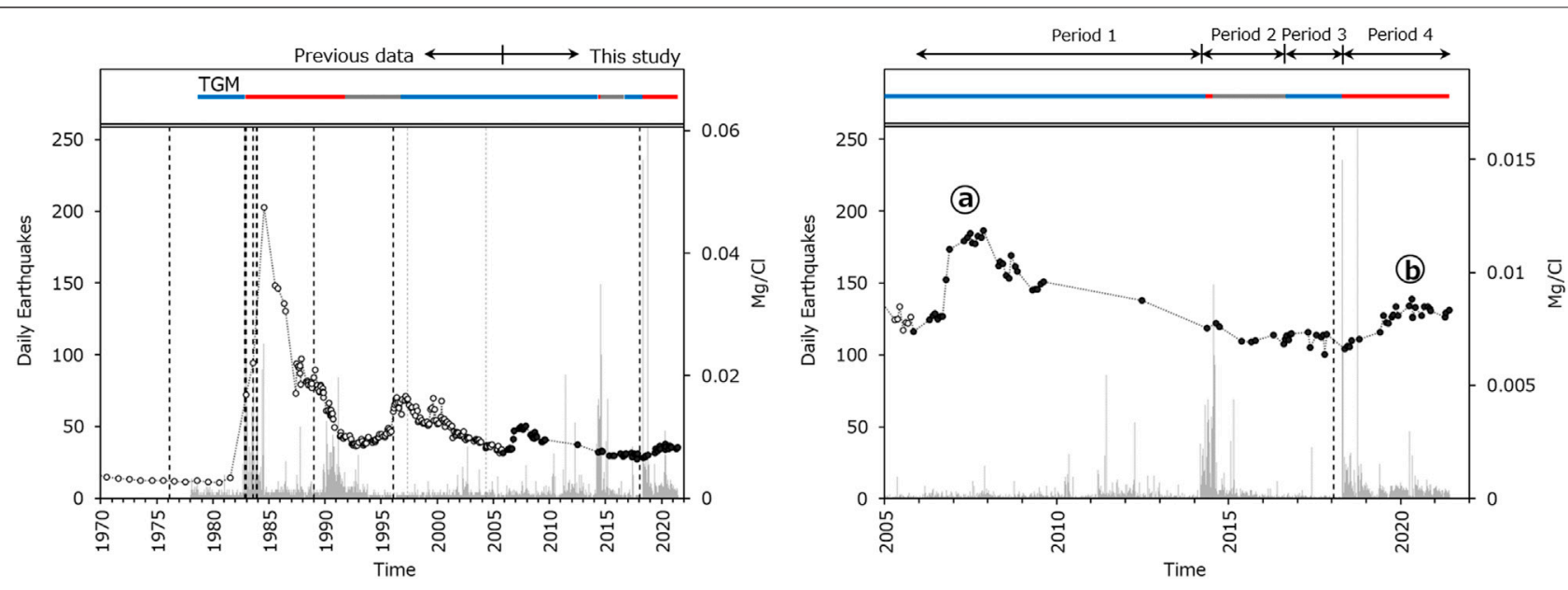

FIGURE 7 | Mg/Cl weight ratio of lake water in Yugama crater. Open and solid circles indicate the previous data since the 1970s (Ohba et al., 2008a) and the present analyses, respectively. TGM and the vertical dashed line show the same as Figure $\mathbf{3}$.

TABLE 3 | Constituent minerals in the ejecta of the 1982-83 eruptions at Yugama Crater Lake (data cited from Ossaka et al. (1984).

\begin{tabular}{|c|c|c|c|c|c|c|c|c|c|c|c|}
\hline Eruption date & Qz & $\mathrm{Cr}$ & $\operatorname{Tr}$ & $\mathbf{F d}$ & Gy & Ah & Al & Py & $\mathrm{Pr}$ & $\mathbf{K a}$ & Mo \\
\hline October 26, 1982 & ++ & + & + & $(+)$ & ++ & - & + & ++ & ++ & ++ & ++ \\
\hline December 29, 1982 & + & + & + & $(+)$ & +++ & - & + & ++ & ++ & +++ & +++ \\
\hline December 29, 1982 & ++ & + & ++ & - & ++ & - & + & ++ & +++ & +++ & + \\
\hline December 29, 1982 & - & + & + & - & - & + & ++ & ++ & ++ & ++ & - \\
\hline July 26, 1983 & + & - & + & $(+)$ & ++ & + & ++ & ++ & ++ & ++ & + \\
\hline November 13, 1983 & ++ & + & + & - & + & - & + & ++ & ++ & ++ & ++ \\
\hline November 13, 1983 & ++ & + & + & - & + & - & + & ++ & ++ & ++ & ++ \\
\hline November 13, 1983 & ++ & + & + & - & + & - & + & ++ & ++ & ++ & $(+)$ \\
\hline November 13, 1983 & ++ & + & + & - & - & - & + & - & + & ++ & $(+)$ \\
\hline
\end{tabular}

Abundance: +++, very abundant; ++, abundant; +, present; -, absent; (+), unidentified.

Qz, Quartz; Cr, Cristobalite; Tr, Tridymite; Fd, Feldspar; Gy, Gypsum; Ah, Anhydrite; Al, Alunite; Py, Pyrite; Pr, Pyrophyllite; Ka, Kaolin; Mo Montmorillonite.

Kakkonda geothermal field, have demonstrated deep groundwater percolation reaching 1,500-3,000 m.b.s. (Saishu et al., 2014; Yaguchi et al., 2016). In the case of a hydrothermal system beneath the Yugama crater, such deep groundwater percolation corresponds to a depth below the self-sealing zone and is sufficient for the groundwater to interact with the mineral deposition of the self-sealing zone, or with the hot plastic rock region.

\section{Period 2 (March 2014-August 2016) $\mathrm{HCl}$ Input Into the Hydrothermal Reservoir}

The earthquake swarm in 2014 was accompanied by a significant increase in $\mathrm{Cl}$ concentration and a decrease in $\mathrm{pH}$, which is similar to the case of the earthquake swarm in 1990-93 (Figure 4) caused by the supply of $\mathrm{HCl}$-rich magmatic fluids from the hot plastic rock region (Ohba et al., 2008a). The state of the magma-hydrothermal system in this period is represented in Figure $6 \mathrm{~B}_{\mathrm{II}}$.

To be precise, acidic gases, such as $\mathrm{HF}, \mathrm{HCl}$, and sulfur gases likely cause decreases in $\mathrm{pH}$ in the crater lake. However, it is difficult to estimate the contribution of each of the acidic gases quantitatively because our analyses (ion chromatography) contain a large uncertainty in the HF concentrations due to an interference by coexisting species. Dissolved $\mathrm{SO}_{4}$ is also not always representative of the total amount of sulfur gases supplied from magma to the crater lake, as explained above. Moreover, $\mathrm{SO}_{2}$ is probably already released for being less soluble in magma with respect to $\mathrm{HCl}$ and $\mathrm{HF}$. The crystallizing magma is hence relatively rich in $\mathrm{HCl}$ with respect to a fresh, less degassed magma (richer in $\mathrm{SO}_{2}$ ). This also explains why the $\mathrm{pH}$ is not lower at Yugama, as for Poás, White Island or Kawah Ijen for instance. Despite the uncertainty in the contributions of $\mathrm{HF}$ and sulfur species, a high correlation was observed between $\mathrm{H}^{+}$and $\mathrm{Cl}$ concentrations during this study period (Figure 8), indicating that the increase in $\mathrm{Cl}$ in 2014-16 was caused by the input of $\mathrm{HCl}$-rich fluids to the hydrothermal reservoir. The slope lower than 1 in the regression line (appears to be $\mathrm{H}^{+}$poor) is probably the result of the suppression of $\mathrm{H}^{+}$activity due to the high ionic strength of the lake water. This is supported by the activity coefficient of $\mathrm{H}^{+}$in the ionic strength of the lake water during this study period $(0.09-0.17)$ obtained by using 
the extended Debye-Hückel equation resulting in $0.81-0.83$, which is very similar to the slope (0.867) of the regression line. These situations were similar to 1990-93; however, the $\delta \mathrm{D}$ and $\delta^{18} \mathrm{O}$ of the lake water after the 2014 activity were the highest ever observed (-39.4\%o and $-1.7 \%$ on August 7, 2016, respectively; Table 2). We propose that the amount of $\mathrm{HCl}-$ rich magmatic fluids in 2014-16 was more significant in magnitude than that in 1990-93. Incidentally, although the main water source of any crater lake is generally meteoric precipitation, Yugama Crater Lake water is more enriched in $\delta \mathrm{D}$ and $\delta^{18} \mathrm{O}$ than the local meteoric water, and the $\delta \mathrm{D} / \delta^{18} \mathrm{O}$ slope is 4.7 (Figure 9A). This slope very much resembles the slope of about 5 , which typically results from the evaporation of lake water at ambient temperature (e.g., Matsubaya and Sakai, 1978; Rowe, 1994; Balistrieri et al., 2007). According to Ohba et al. (2000), however, this isotopic enrichment mainly results from evaporation, but there is a small contribution of isotopically heavy $\mathrm{Cl}$-rich fluids. This isotopically heavy fluids is a liquid phase separated by boiling of a mixture of local meteoric water and a magmatic fluids rich in $\mathrm{Cl}$. The isotopic enrichment of lake water relative to local meteoric water $\left(\Delta \delta \mathrm{D}\right.$ and $\left.\Delta \delta^{18} \mathrm{O}\right)$ can be obtained by subtracting the $\delta$ value of local meteoric water $\left(\delta \mathrm{D}_{\mathrm{lmw}}=-79.3 \%\right.$ and $\delta^{18} \mathrm{O}_{\mathrm{lmw}}=$ $-11.8 \%$; Ohba et al., 2000) from the $\delta$ value of lake water $\left(\delta \mathrm{D}_{\text {lake }}\right.$ and $\left.\delta^{18} \mathrm{O}_{\text {lake }}\right)$. For example, the $\Delta \delta$ values on August 7 , 2016, are calculated as $\Delta \delta \mathrm{D}=+39.9 \%$ ond $\Delta \delta^{18} \mathrm{O}=+10.1 \%$. On the other hand, the isotope enrichment of lake water by the addition of isotopically heavy Cl-rich fluids $\left(\Delta \delta \mathrm{D}_{\mathrm{Cl}}\right.$ and $\left.\Delta \delta^{18} \mathrm{O}_{\mathrm{Cl}}\right)$ can be estimated by multiplying the $\mathrm{Cl}$ concentration $(\mathrm{mg} / \mathrm{L})$ of the lake water by the coefficients, $2.10 \times 10^{-3}$ for $\delta \mathrm{D}$ and $5.16 \times 10^{-4}$ for $\delta^{18} \mathrm{O}$ (see Ohba et al., 2000 for more details). Based on this simple calculation, it is estimated that the $\Delta \delta \mathrm{D}_{\mathrm{Cl}}=+10.9 \%$ and $\Delta \delta^{18} \mathrm{O}_{\mathrm{Cl}}=+2.7 \%$, which account for 27.3 and $26.7 \%$ of $\Delta \mathrm{D}$ and $\Delta^{18} \mathrm{O}$, respectively. The contributions of the addition of isotopically heavy $\mathrm{Cl}$-rich fluids to total isotope enrichment calculated in this way were $21-32 \%$ (average in $\delta \mathrm{D}$ and $\delta^{18} \mathrm{O}$ ) throughout the present study period, which is not significantly different from the period 1990-93 (Figure 9B). In other words, although there is isotope enrichment by addition of $\mathrm{Cl}$-rich fluids, the heavy isotope ratio of lake water relative to local meteoric water is mainly due to evaporative enrichment even during this study period.

\section{Self-Sealing Zone Breach due to Magmatic Fluids Ascend}

In addition to $\mathrm{Cl}$ and $\mathrm{SO}_{4}$, the concentrations of rock-forming species increased, especially $\mathrm{Ca}, \mathrm{Al}, \mathrm{Fe}$, and $\mathrm{Si}$ (Figure 5). As mentioned above, these species are probably derived from the secondary mineral deposition. The increases in these components suggest that a breach (dissolution) of the self-sealing zone occurred corresponding to the earthquake swarm in 2014. However, we believe that the breaching process was different than the one in 2006.

The significant increase in $\mathrm{Cl}$ suggests that the breach of the self-sealing zone in 2014 was caused by an internal pressurization due to the Cl-rich fluids accumulation or

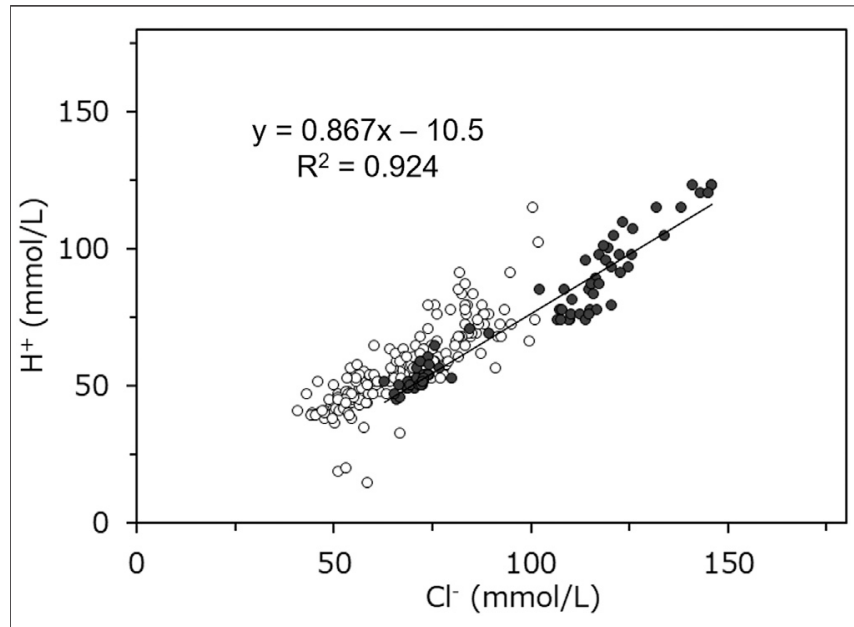

FIGURE 8 | Relationship between $\mathrm{H}^{+}$and $\mathrm{Cl}$ concentrations. $\mathrm{H}^{+}$ concentrations were calculated as $\left[\mathrm{H}^{+}\right]=10^{-\mathrm{pH}}$. Open and solid circles indicate the previous data since the 1970s (Ohba et al., 2008a) and the present analyses, respectively. The regression line is calculated for the lake water during this study period (November 2005 to May 2021; Table 2).

injection from depth. The deep deformation source pressurized in 2013-2015 (at a depth of $6.3 \mathrm{~km}$ and $2 \mathrm{~km}$ northwest of the Yugama Crater Lake; Munekane, 2021) may not be a direct source of $\mathrm{Cl}$ because the $\mathrm{HCl}$ should remain dissolved in the magma at this depth (e.g., Christopher et al., 2015), but may have induced the supply of Cl-rich magma fluids from the crystallizing magma. During this period, there were no obvious increases in the $\mathrm{Mg} / \mathrm{Cl}$ ratio despite the breach of the self-sealing zone (Figure 7). This implies that the ascending of the Cl-rich magmatic fluids from inside the self-sealing zone through the breached section inhibited the deep circulation of the groundwater; as a result, the groundwater could not intrude into the hot plastic rock region inside the self-sealing zone (path "ii" in Figure 6B $\mathrm{B}_{\mathrm{II}}$ ).

All eruptions at the Yugama crater since 1982 were accompanied by an increase in the $\mathrm{Mg} / \mathrm{Cl}$ ratio during a decrease in $\mathrm{Cl}$ concentration (Figure 5A). In contrast, neither eruptions nor increases in $\mathrm{Mg} / \mathrm{Cl}$ ratio occurred in 1990 and 2014, when a significant $\mathrm{HCl}$ input occurred (Figure 7). This means that the contact between groundwater and the hot plastic rocks has played an important role in triggering phreatic eruptions at the Yugama crater since 1982, rather than the magmatic fluids input to the hydrothermal reservoir. In other words, fresh magma or actively degassing volcanic systems are not necessarily required to trigger phreatic eruptions. The water temperature in the Yugama Crater Lake has remained relatively cold for decades, although it once reached $55.5^{\circ} \mathrm{C}$ just after the eruption in 1982 (Tokyo Institute of Technology et al., 1983), compared to the hyperacid lake of Poás volcano (up to $94^{\circ} \mathrm{C}$; Rowe, 1994), where magmatic or phreatomagmatic eruptions occur. This seems to support the idea that the contact between groundwater and the hot 

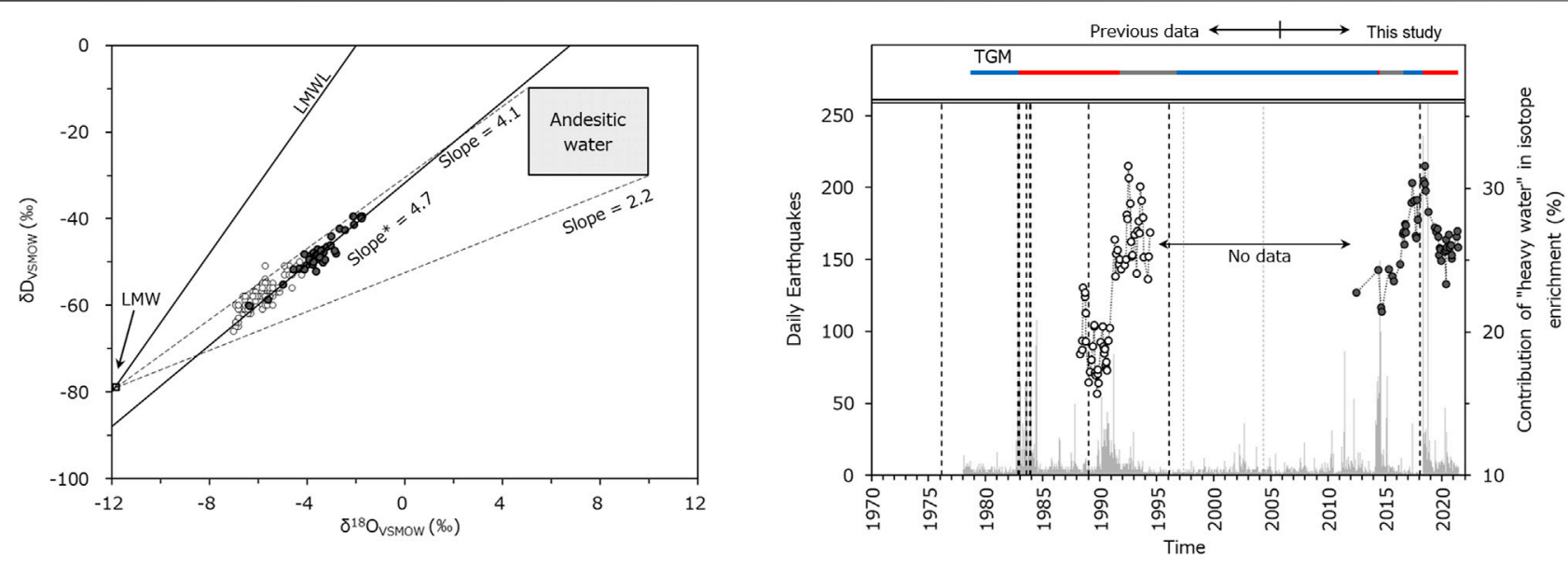

FIGURE 9 | (A) $\delta$ D vs. $\delta^{18} \mathrm{O}$ plot for lake water in Yugama crater. The "andesitic water" field $\left(\delta \mathrm{D}=-10\right.$ to $-30 \%$ o, $\delta^{18} \mathrm{O}=+5$ to $+10 \%$; Taran et al., 1989 ; Giggenbach., 1992b), local meteoric water (LMW, $\delta \mathrm{D}=-79 \%$, $\delta^{18} \mathrm{O}=-11.8 \%$; Ohba et al., 2000), and the local meteoric water line (LMWL, $\delta \mathrm{D}=8 \delta^{18} \mathrm{O}+16$; Ohwada et al., 2003$)$ are also shown. ${ }^{*} T h e, \delta \mathrm{D} / \delta^{18} \mathrm{O}$ slope (4.7) for the lake water during this study period. (B) The contribution of "heavy water" addition in the isotopic enrichment of lake water (average in $\delta \mathrm{D}$ and $\delta^{18} \mathrm{O}$ ). Open and solid circles indicate the previous data (available from 1988 to 1996; Ohba et al., 2000) and the present analyses, respectively.

plastic rocks, which triggered the recent phreatic eruptions at Yugama Crater Lake, was not caused by magma intrusion, or magma intrusions at Yugama Crater Lake might be fairly small in volume with no critical mass to destabilize the hydrothermal system and trigger eruptions.

\section{Period 3 (September 2016-March 2018) Regrowth of the Self-Sealing Zone}

The changes in water chemistry in the Yugama Crater Lake during this period are characterized by an increase (recovering) in $\mathrm{pH}$ and a general decrease in the concentrations of various components (Figures 4, 5). Considering the low seismic activity and cooling in the hydrothermal reservoir suggested by the magnetizing trend, we suggest that the self-sealing zone was redeveloped during this recovery period, leading to the suppression of the ascending magmatic fluids to the Yugama crater (Figure 6A). According to the volcanic gas study (Ohba et al., 2019b), the redevelopment of the self-sealing zone has been suggested as the decrease in the fraction of magmatic components in the volcanic gas around the Yugama Crater Lake from April 2016 to November 2017, which is consistent with the interpretation of the present study. This situation is similar to Period 1, but because this period ended rapidly, the state of the magma-hydrothermal system did not move from the dynamics exposed in Figures 6A-6B $\mathbf{I}$, as in Period 1.

\section{Period 4 (April 2018-May 2021) Intermittent Breaching of the Self-Sealing Zone due to Less Intense Ascent of Magmatic Fluids}

Increases in $\mathrm{Ca}, \mathrm{Al}, \mathrm{Fe}, \mathrm{Si}$, and $\mathrm{Cl}$, an earthquake swarm, and a repressurizing of the deep source (Munekane, 2021) were observed. Based on the similarity to the event in 2014, we believe that the magmatic fluid intermittently breached the self-sealing zone, causing repeated injections of the magmatic fluid to the Yugama crater. Although the number of daily earthquakes in 2018 (324 earthquakes on September 29) is larger than that in 2014 (149 on July 24), the lake water temperature, $\mathrm{Cl}$ concentration, $\delta \mathrm{D}$, and $\delta^{18} \mathrm{O}$ values since 2018 are lower than those observed in 2014 (Figure 5; Table 2). The amount of magmatic fluids supply in 2018-21 was probably smaller than in 2014-16, which is consistent with the slight increase in the $\mathrm{Mg} / \mathrm{Cl}$ ratio in 2018 (circle " $\mathrm{b}$ " in Figure 7). Because of the less intense discharge of magmatic fluids from the self-sealing zone, groundwater intruded into the hot plastic rock region inside the self-sealing zone, allowing the transport of $\mathrm{Mg}$ to the Yugama crater (path "iii" in Figure $\left.6 B_{I I I}\right)$.

\section{Aging of Crystallizing Magma}

Although we should be cautious because the total amount of $\mathrm{S}$ was not analyzed in this study, the long-term $\mathrm{SO}_{4} / \mathrm{Cl}$ molar ratio fluctuates along a general decreasing trend (Figure 10A). Sulfur is one of the major volatiles in magma, but the magma becomes relatively $\mathrm{S}$-poor, $\mathrm{Cl}$-rich as degassing progresses because $\mathrm{SO}_{2}$ is less soluble than $\mathrm{HCl}$ (Giggenbach, 1996). The decrease in the $\mathrm{S} / \mathrm{Cl}$ ratio with progressive degassing has been demonstrated in volcanic gases. At Unzen volcano in Japan, where magmatic eruptions occurred from 1991 to 1996, the S/Cl ratio of volcanic gases collected from the cooling lava dome after the eruption (Shinohara et al., 2008) was lower than the $\mathrm{S} / \mathrm{Cl}$ ratio detected during the eruption (Ohba et al., 2008b). Therefore, the long-term decrease in the $\mathrm{S} / \mathrm{Cl}$ ratio in the lake water may indicate that the crystallizing magma beneath the Yugama crater is gradually becoming $\mathrm{S}$-depleted due to the progress of degassing. On the other hand, the lake water analyzed in this study has a lower $\mathrm{SO}_{4}$ concentration and a higher $\mathrm{Cl}$ concentration than in the previous studies (before November 2005; Figure 10B), indicating that the decreasing trend of the $\mathrm{SO}_{4} / \mathrm{Cl}$ ratio of the lake water is not only due to sulfur depletion but also to the 

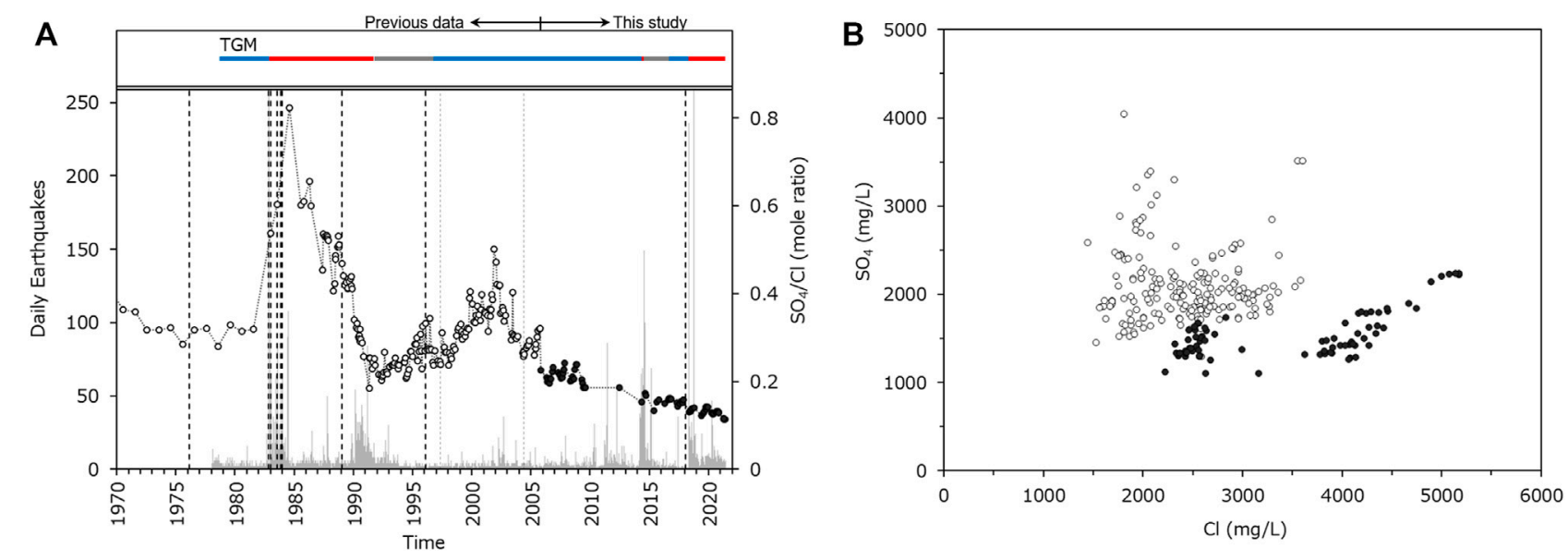

FIGURE 10| (A) The $\mathrm{SO}_{4} / \mathrm{Cl}$ mole ratio of lake water in Yugama crater. Data before October 2005 were cited from Ohba et al. (2008a). TGM and the vertical dashed line show the same as Figure 3. (B) $\mathrm{SO}_{4}$ Vs. Cl plot for lake water in Yugama crater. Open and solid circles indicate the previous data since the 1970s (Ohba et al., $2008 \mathrm{a}$ ) and the present analyses, respectively.

increase in $\mathrm{Cl}$ concentration. According to Bodnar (1992), the salinity of brine exsolving from a magma crystallizing below $1.3 \mathrm{kbar}$ is initially low, but it continues to increase as the magma crystallizes and eventually exceeds $50 \mathrm{wt} \%$. That is, the increase in $\mathrm{Cl}$ concentration can be interpreted as being caused by the progress of magma crystallization, and this is responsible for the decrease in the $\mathrm{SO}_{4} / \mathrm{Cl}$ ratio of the lake water along with the sulfur depletion.

In addition, it is interesting that the peak value of the $\mathrm{Mg} / \mathrm{Cl}$ ratio in Yugama Crater Lake decreases with age (Figure 7). We believe that the hot plastic rock region may be shrinking during the repeated cycles of breach and regrowth of the selfsealing zone (Figure $\mathbf{6} \mathbf{A}^{\prime}$ ). However, this hypothesis does not guarantee that the $\mathrm{SO}_{4} / \mathrm{Cl}$ and $\mathrm{Mg} / \mathrm{Cl}$ ratios in the Yugama crater will continue to decrease in the future. If new magma intrusions, extrusions, or exposures of hot plastic rock regions occur in the future, these indicators should be refreshed. Therefore, lake water monitoring should continue. Also, phreatic eruptions triggered by other mechanisms, such as eruptions involving the self-sealing of the liquid sulfur pool at the lake bottom (Rouwet and Morrissey, 2015; Rouwet et al., 2017), should be focused on for future safety and research because a molten sulfur pool has been found at the bottom of the Yugama Crater Lake.

\section{CONCLUSION}

Water samples collected from Yugama Crater Lake at KusatsuShirane volcano were analyzed from November 2005 to May 2021 to discuss the magma-hydrothermal interaction beneath this crater lake and a key mechanism for the recent eruptions at this crater. The conclusions drawn are summarized as follows.
1) The magma beneath the Yugama crater supplies $\mathrm{HCl}$-rich fluid to the lake through the hydrothermal reservoir. The long-term $\mathrm{SO}_{4} / \mathrm{Cl}$ ratio in Yugama Crater Lake is decreasing, which may reflect the chemical evolution of magmatic fluid due to the degassing process of crystallizing magma.

2) Suppression of magmatic fluids due to the development of the self-sealing zone surrounding degassing magma was estimated from 2005 to 2012 because $\mathrm{Cl}$ and $\mathrm{SO}_{4}$ concentrations in lake water decreased slowly during this period. On the other hand, increases in certain rock-forming species, including $\mathrm{Ca}, \mathrm{Si}$, and $\mathrm{Al}$, as well as in the $\mathrm{Mg} / \mathrm{Cl}$ ratio were observed from 2006 to 2007. This is because the suppression of ascending magmatic fluids due to the developed self-sealing zone led to the deep circulation of groundwater, the chemical and arguably physical leaching of the self-sealing zone, and, eventually, the contact with the hot plastic rock region.

3) An input of magmatic fluid to the hydrothermal reservoir breaching the self-sealing zone was estimated in 2014 because a significant increase in $\mathrm{Cl}$ concentrations and those of certain solute metal species indicating the leaching of the self-sealing zone, such as $\mathrm{Ca}, \mathrm{Al}, \mathrm{Fe}$, and $\mathrm{Si}$, were observed in lake water. At this time, the $\mathrm{Mg} / \mathrm{Cl}$ ratio of the lake water did not increase because the ascending magmatic fluid inhibited the deep intrusion of groundwater into the hot plastic rock region.

4) The input of magmatic fluid through the self-sealing zone was repeated in 2018, 2019, and 2020 on a smaller scale than after the 2014 earthquakes, shown by only slight increases in the $\mathrm{Mg} / \mathrm{Cl}$ ratio, suggesting the less intense ascend of magmatic fluid, allowing deep groundwater intrusion to the hot plastic rock region.

5) The $\delta \mathrm{D}$ and $\delta^{18} \mathrm{O}$ of the waters in the Yugama crater lake are enriched relative to the local meteoric water. This is mainly due to evaporation effects, but magmatic fluid input accounts for $21-32 \%$ (average in $\delta \mathrm{D}$ and $\delta^{18} \mathrm{O}$ ) of the total isotopic 
enrichment relative to local meteoric water throughout the present study period.

6) The contact between the groundwater and the hot plastic rock played an essential role in the mechanism of the eruptions that occurred at the Yugama crater after 1982, rather than the input of magmatic gas itself. This is because these eruptions were always accompanied by an increase in the $\mathrm{Mg}$ concentration and the $\mathrm{Mg} / \mathrm{Cl}$ ratio, and they occurred during a decrease in the $\mathrm{Cl}$ concentration, while the $\mathrm{Mg} / \mathrm{Cl}$ ratio did not increase and no eruptions occurred during the 1990 and 2014 unrest when there were significant $\mathrm{HCl}$ supplies to the Yugama crater.

Our study demonstrates that, in contrast to more actively degassing volcanic systems which are involved in the eruptions at hyperacid crater lakes, such as Poás and Kawah Ijen, phreatic eruptions can potentially occur without clear signs of new magma intrusions, but also during stages of cooling of relatively degassed magmas.

\section{DATA AVAILABILITY STATEMENT}

The original contributions presented in the study are included in the article/Supplementary Material, further inquiries can be directed to the corresponding author.

\section{REFERENCES}

Badrudin, M. (1994). Kelut Volcano Monitoring: Hazards, Mitigation and Changes in Water Chemistry Prior to the 1990 Eruption. Geochem. J. 28, 233-241. doi:10.2343/geochemj.28.233

Balistrieri, L. I., Shanks, W. C., Cuhel, R. L., Aguilar, C., and Klump, J. V. (2007). "The Influence of Sublacustrine Hydrothermal Vent Fluids on the Geochemistry of Yellowstone Lake," in Integrated Geoscience Studies In the Greater Yellowstone Area-Volcanic, Tectonic, and Hydrothermal Processes In the Yellowstone Geoecosystem. Editor L. A. Morgan (Reston, Virginia: U.S. Geological Survey), 169-199.

Barberi, F., Bertagnini, A., Landi, P., and Principe, C. (1992). A Review on Phreatic Eruptions and Their Precursors. J. Volcanology Geothermal Res. 52, 231-246. doi:10.1016/0377-0273(92)90046-G

Bernard, A., and Mazot, A. (2004). "Geochemical Evolution of the Young Crater lake of Kelud Volcano in Indonesia," in Water-Rock Interaction (WRI-11). Editors R. B. Wanty and R. R. Seal (Leiden; New York: A.A. Balkema), 87-90.

Bodnar, R. J. (1992). Can We Recognize Magmatic Fluid Inclusions in Fossil Systems Based on Room-Temperature Phase Relations and Microthermometric Behavior? Rept. Geol. Surv. Jpn. 279, 26-30.

Browne, P. R. L., and Lawless, J. V. (2001). Characteristics of Hydrothermal Eruptions, with Examples from New Zealand and Elsewhere. Earth-Science Rev. 52, 299-331. doi:10.1016/S0012-8252(00)00030-1

Caudron, C., Lecocq, T., Syahbana, D. K., McCausland, W., Watlet, A., Camelbeeck, T., et al. (2015). Stress and Mass Changes at a "wet" Volcano: Example during the 2011-2012 Volcanic Unrest at Kawah Ijen Volcano (Indonesia). J. Geophys. Res. Solid Earth 120, 5117-5134. doi:10.1002/ 2014JB011590

Christenson, B. W., Reyes, A. G., Young, R., Moebis, A., Sherburn, S., Cole-Baker, J., et al. (2010). Cyclic Processes and Factors Leading to Phreatic Eruption Events: Insights from the 25 September 2007 Eruption through Ruapehu Crater Lake, New Zealand. J. Volcanology Geothermal Res. 191, 15-32. doi:10.1016/ j.jvolgeores.2010.01.008

\section{AUTHOR CONTRIBUTIONS}

MY drafted the manuscript. MY and TO sampled lake water by using a ladle and then analyzed the samples. AT sampled lake water by using a ladle and a drone. All authors read and approved the final manuscript.

\section{FUNDING}

This study was supported by ERI JURP (2020-KOBO11, 2019-Yvolcano5, 2018-Y-volcano4, 2017-Y-volcano4) in Earthquake Research Institute, the University of Tokyo, and the Japanese Ministry of Education, Culture, Sports, Science and Technology, under grant of the Integrated Program for Next Generation Volcano Research and Human Resource Development 2016 to 2021 .

\section{ACKNOWLEDGMENTS}

We wish to express our sincere appreciation to S. Matsusue of JMA for providing the information on daily earthquakes and the funders for their financial support of this study, the two reviewers (YT and MM), the handling editor (DR), and the chief editor (Valerio Acocella) for their efforts in reviewing and providing constructive comments to improve the manuscript.

Christenson, B. W., and Wood, C. P. (1993). Evolution of a Vent-Hosted Hydrothermal System beneath Ruapehu Crater Lake, New Zealand. Bull. Volcanol. 55, 547-565. doi:10.1007/BF00301808

Christopher, T. E., Blundy, J., Cashman, K., Cole, P., Edmonds, M., Smith, P. J., et al. (2015). Crustal-scale Degassing Due to Magma System Destabilization and Magma-Gas Decoupling at Soufrière Hills Volcano, Montserrat. Geochem. Geophys. Geosyst. 16, 2797-2811. doi:10.1002/2015GC005791

Fournier, R. O. (1999). Hydrothermal Processes Related to Movement of Fluid from Plastic into Brittle Rock in the Magmatic-Epithermal Environment. Econ. Geol. 94, 1193-1211. doi:10.2113/gsecongeo.94.8.1193

Giggenbach, W. F. (1996). "Chemical Composition of Volcanic Gases," in Monitoring And Mitigation Of Volcano Hazards. Editors R. Scarpa and R. I. Tilling (Berlin: Springer), 221-256. doi:10.1007/978-3-64280087-0_7

Giggenbach, W. F., and Glover, R. B. (1975). The Use of Chemical Indicators in the Surveillance of Volcanic Activity Affecting the Crater Lake on Mt Ruapehu, New Zealand. Bull. Volcanol. 39, 70-81. doi:10.1007/BF02596947

Giggenbach, W. F. (1992b). Isotopic Shifts in Waters from Geothermal and Volcanic Systems along Convergent Plate Boundaries and Their Origin. Earth Planet. Sci. Lett. 113, 495-510. doi:10.1016/0012-821X(92) 90127-H

Giggenbach, W. F. (1992a). Magma Degassing and Mineral Deposition in Hydrothermal Systems along Convergent Plate Boundaries. Econ. Geol. 87, 1927-1944. doi:10.5382/SP.10.01

Hayakawa, Y., and Yui, M. (1989). Eruptive History of the Kusatsu Shirane Volcano. Daiyonki-kenkyu 28, 1-17. doi:10.4116/jaqua.28.1

Hedenquist, J. W., and Henley, R. W. (1985). Hydrothermal Eruptions in the Waiotapu Geothermal System, New Zealand; Their Origin, Associated Breccias, and Relation to Precious Metal Mineralization. Econ. Geol. 80, 1640-1668. doi:10.2113/gsecongeo.80.6.1640

Ida, Y., Osada, N., Sawada, M., Koyama, E., and Kagiyama, T. (1989). Seismological Study Based on Recently Installed Permanent Stations and a Small Eruptive Event on January 6, 1989 at Kusatsu-Shirane Volcano. Bull. Earthq. Res. Inst. Univ. Tokyo 64, 325-345. doi:10.15083/0000032805 
Ishizaki, Y., Nigorikawa, A., Kametani, N., Yoshimoto, M., and Terada, A. (2020). Geology and Eruption History of the Motoshirane Pyroclastic Cone Group, Kusatsu-Shirane Volcano, central Japan. Jour. Geol. Soc. Jpn. 126, 473-491. doi:10.5575/geosoc.2020.0022

Japan Meteorological Agency (2013). "Kusatsu-Shiranesan," in National Catalogue of the Active Volcanoes in Japan. 4th Edition (Tokyo: Japan Meteorological Agency), 44645-44672. Available at: https://www.data.jma.go.jp/svd/vois/data/ tokyo/STOCK/souran_eng/volcanoes/044_kusatsu-shiranesan.pdf (Accessed July 14, 2021).

Japan Meteorological Agency (2021). Seismology and Volcanology Department, JMA. Available at: http://www.jma.go.jp/jma/press/2106/30a/yochiren210630_ 1.pdf (Accessed July 14, 2021).

Japan Meteorological Agency (2018). Volcanic Activity of Kusatsu-Shiranesan Volcano (January 2017-June 8, 2017). Rep. Coord. Comm. Predict. Volcan. Eruption 127, 53-68. (in Japanese).

Japan Meteorological Agency (2019). Volcanic Activity of Kusatsu-Shiranesan Volcano (October 2018-January 2019). Rep. Coord. Comm. Predict. Volcan. Eruption 132, 67-92. (in Japanese).

Kakioka Magnetic Observatory, JMA (2016). Variation of Geomagnetic Total Intensity at Kusatsu-Shirane Volcano. Rep. Coord. Commit. Pred. Volc. Eruption 118, 22-23. (in Japanese).

Kakioka Magnetic Observatory, JMA (2019). Variation of Geomagnetic Total Intensity at Kusatsu-Shirane Volcano. Rep. Coord. Commit. Pred. Volc. Eruption 132, 93-96. (in Japanese).

Kametani, N., Ishizaki, Y., Katsuoka, N., Yoshimoto, M., and Terada, A. (2021). Eruption Styles and Ages of the Shirane-Minami Crater Chain and the YumiIke Maar on the Southern Foot of the Shirane Pyroclastic Cone Group, KusatsuShirane Volcano, Central Japan. Bull. Volcanol. Soc. Jpn. 66, 1-19. doi:10.18940/ kazan.66.1_1

Kaneko, T., Shimizu, S., and Itaya, T. (1991). K-ar Ages of the Quaternary Volcanoes in the Shin-Etsu highland Area, central Japan, and Their Formation History. Bull. Earthq. Res. Inst. Univ. Tokyo 66, 299-332. doi:10.15083/0000032764

Kasai, K., Sakagawa, Y., Komatsu, R., Sasaki, M., Akaku, K., and Uchida, T. (1998). The Origin of Hypersaline Liquid in the Quaternary Kakkonda Granite, Sampled from Well WD-1a, Kakkonda Geothermal System, Japan. Geothermics 27, 631-645. doi:10.1016/S0375-6505(98)00037-6

Komatsu, R., Ikeuchi, K., Doi, N., Sasaki, M., Uchida, T., and Sasada, M. (1998). Characteristics of the Quaternary Kakkonda Granite and Geothermal System Clarified by Fluid Inclusion Study of Deep Investigation Well, Kakkonda, Japan. J. Geotherm. Res. Soc. Jpn. 20, 209-224. doi:10.11367/grsj1979.20.209

Kusatsu-Shirane Volcano Observatory, Tokyo Institute of Technology (2021). "Crustal Deformation Around the Yugama Crater of Kusatsu Shirane Volcano*," in The 148th Coordinating Committee for Prediction of Volcanic Eruption: Part 1-1 Kusatsu-Shirane Volcano (Tokyo: Japan Meteorological Agency), 19-20.

Kusatsu-Shirane Volcano Observatory, Tokyo Institute of Technology (1996). The Activity on February, 1996 of Kusatsu-Shirane Volcano. Rep. Coord. Commit. Pred. Volc. Eruption 65, 29-32. (in Japanese).

Kusatsu-Shirane Volcano Observatory, Tokyo Institute of Technology (1997). The Occurrence of Sudden Gas Jet and Water Dome at Yugama Crater on 2nd May, 1997. Rep. Coord. Commit. Pred. Volc. Eruption 68, 33-34. (in Japanese).

Mannen, K., Roman, D., Leonard, G., Prejean, S., and Nakagawa, M. (2019). Special Issue "Towards Forecasting Phreatic Eruptions: Examples from Hakone Volcano and Some Global Equivalents". Earth Planets Space 71, 91. doi:10.1186/s40623-019-1068-9

Martínez, M., Fernández, E., Valdés, J., Barboza, V., Van derLaat, R., Duarte, E., et al. (2000). Chemical Evolution and Volcanic Activity of the Active Crater lake of Poás Volcano, Costa Rica, 1993-1997. J. Volcanol. Geotherm. Res. 97, 127-141. doi:10.1016/S0377-0273(99)00165-1

Martínez, M. (2008). Geochemical Evolution of the Acid Crater lake of Poás Volcano (Costa Rica): Insights into Volcanic-Hydrothermal Processes. Netherlands (Utrecht): Universiteit Utrecht. Ph.D. thesis.

Martínez, M., van Bergen, M. J., Takano, B., Fernández-Soto, E., and BarqueroHernández, J. (2019). "Behaviour of Polythionates in the Acid Lake of Poás Volcano: Insights into Changes in the Magmatic-Hydrothermal Regime and Subaqueous Input of Volatiles," in Poás Volcano, Active Volcanoes of the World.
Editors F. Tassi, O. Vaselli, and R. Mora-Amador (Cham: Springer), 155-202. doi:10.1007/978-3-319-02156-0_7

Mastin, L. G. (1995). Thermodynamics of Gas and Steam-Blast Eruptions. Bull. Volcanol. 57, 85-98. doi:10.1007/BF00301399

Matsubaya, O., and Sakai, H. (1978). D/H and 18O/16O Fractionation Factors in Evaporation of Water at 60 and 80.DEG.C. Geochem. J. 12, 121-126. doi:10.2343/geochemj.12.121

Matsunaga, Y., Kanda, W., Takakura, S., Koyama, T., Saito, Z., Seki, K., et al. (2020). Magmatic Hydrothermal System Inferred from the Resistivity Structure of Kusatsu-Shirane Volcano. J. Volcanology Geothermal Res. 390, 106742. doi:10.1016/j.jvolgeores.2019.106742

Munekane, H. (2021). Modeling Long-Term Volcanic Deformation at KusatsuShirane and Asama Volcanoes, Japan, Using the GNSS Coordinate Time Series. Earth Planets Space 73, 192. doi:10.1186/s40623-021-01512-2

Muraoka, H., Uchida, T., Sasada, M., Yagi, M., Akaku, K., Sasaki, M., et al. (1998). Deep Geothermal Resources Survey Program: Igneous, Metamorphic and Hydrothermal Processes in a Well Encountering $500^{\circ} \mathrm{C}$ at 3729 M Depth, Kakkonda, japan. Geothermics 27, 507-534. doi:10.1016/S0375-6505(98) 00031-5

Nurhasan, N., Ogawa, Y., Ujihara, N., Tank, S. B., Honkura, Y., Onizawa, S. y., et al. (2006). Two Electrical Conductors beneath Kusatsu-Shirane Volcano, Japan, Imaged by Audiomagnetotellurics, and Their Implications for the Hydrothermal System. Earth Planet Space 58, 1053-1059. doi:10.1186/ BF03352610

Ogawa, Y., Aoyama, H., Yamamoto, M., Tsutsui, T., Terada, A., Ohkura, T., et al. (2018). Comprehensive Survey of 2018 Kusatsu-Shirane Eruption. Proc. Symp. Natural Disaster Sciences 55, 25-30. (in Japanese).

Ohba, T., Hirabayashi, J.-i., Nogami, K., Kusakabe, M., and Yoshida, M. (2008b). Magma Degassing Process during the Eruption of Mt. Unzen, Japan in 1991 to 1995: Modeling with the Chemical Composition of Volcanic Gas. J. Volcanology Geothermal Res. 175, 120-132. doi:10.1016/ j.jvolgeores.2008.03.040

Ohba, T., Hirabayashi, J.-i., and Nogami, K. (2008a). Temporal Changes in the Chemistry of lake Water within Yugama Crater, Kusatsu-Shirane Volcano, Japan: Implications for the Evolution of the Magmatic Hydrothermal System. J. Volcanology Geothermal Res. 178, 131-144. doi:10.1016/ j.jvolgeores.2008.06.015

Ohba, T., Hirabayashi, J.-i., and Nogami, K. (1994). Water, Heat and Chloride Budgets of the Crater lake, Yugama at Kusatsu-Shirane Volcano, Japan. Geochem. J. 28, 217-231. doi:10.2343/geochemj.28.217

Ohba, T., Hirabayashi, J., and Nogami, K. (2000). D/H and 18 O/16 O Ratios of Water in the Crater lake at Kusatsu-Shirane Volcano, Japan. J. Volcanology Geothermal Res. 97, 329-346. doi:10.1016/S03770273(99)00169-9

Ohba, T., Yaguchi, M., Nishino, K., Numanami, N., Daita, Y., Sukigara, C., et al. (2019a). Time Variations in the Chemical and Isotopic Composition of Fumarolic Gases at Hakone Volcano, Honshu Island, Japan, over the Earthquake Swarm and Eruption in 2015, Interpreted by Magma Sealing Model. Earth Planets Space 71, 48. doi:10.1186/s40623-019-1027-5

Ohba, T., Yaguchi, M., Nishino, K., Numanami, N., Tsunogai, U., Ito, M., et al. (2019b). Time Variation in the Chemical and Isotopic Composition of Fumarolic Gasses at Kusatsu-Shirane Volcano, Japan. Front. Earth Sci. 7, 249. doi:10.3389/feart.2019.00249

Ohchi, K. (1987). Observation of the Geomagnetic Total Force at Kusatsu-Shirane Volcano. Mem. Kakioka Mag. Obs. 22, 1-9.

Ohwada, M., Ohba, T., Hirabayashi, J.-i., Nogami, K., Nakamura, K., and Nagao, K. (2003). Interaction between Magmatic Fluid and Meteoric Water, Inferred from $18 \mathrm{O} / 16 \mathrm{O}$ and $36 \mathrm{Ar} / \mathrm{H} 2 \mathrm{O}$ Ratios of Fumarolic Gases at the Kusatsu Shirane Volcano, Japan. Earth Planet Space 55, 105-110. doi:10.1186/BF03351737

Ossaka, J., Hirabayashi, J., and Ozawa, T. (1984). "The Activity of Kusatsu-Shirane Volcano in Recent Years and Geochemical Study ${ }^{*}$," in Fundamental Research for Predicting Volcanic Eruption through Observation of Volcanic Gases. Editor J. Ossaka (Japan: Ministry of Education), 96-112.

Rouwet, D., Mora Amador, R. A., Sandri, L., Ramírez-UmañaGonzález, C. G., González, G., Pecoraino, G., et al. (2019). "39 Years of Geochemical Monitoring of Laguna Caliente Crater Lake, Poás: Patterns from the Past as Keys for the Future," in Poás Volcano, Active Volcanoes of the World. Editors F. Tassi, 
O. Vaselli, and R. Mora-Amador (Cham: Springer), 213-233. doi:10.1007/9783-319-02156-0_9

Rouwet, D., Mora-Amador, R., Ramírez-Umaña, C. J., González, G., and Inguaggiato, S. (2017). Dynamic Fluid Recycling at Laguna Caliente (Poás, Costa Rica) before and during the 2006-ongoing Phreatic Eruption Cycle (2005-10). Geol. Soc. Lond. Spec. Publications 437, 73-96. doi:10.1144/SP437.11

Rouwet, D., and Morrissey, M. M. (2015). "Mechanisms of Crater lake Breaching Eruptions," in Volcanic Lakes, Advances in Volcanology. Editors D. Rouwet, B. Christenson, F. Tassi, and J. Vandemeulebrouck (Heidelberg: Springer), 73-91. doi:10.1007/978-3-642-36833-2_3

Rouwet, D., Tassi, F., Mora-Amador, R., Sandri, L., and Chiarini, V. (2014). Past, Present and Future of Volcanic lake Monitoring. J. Volcanology Geothermal Res. 272, 78-97. doi:10.1016/j.jvolgeores.2013.12.009

Rowe, G. L., Ohsawa, S., Takano, B., Brantley, S. L., Fernandez, J. F., and Barquero, J. (1992). Using Crater Lake Chemistry to Predict Volcanic Activity at Poás Volcano, Costa Rica. Bull. Volcanol. 54, 494-503. doi:10.1007/ BF00301395

Rowe, Jr., G. L. (1994). Oxygen, Hydrogen, and Sulfur Isotope Systematics of the Crater lake System of Poas Volcano, Costa Rica. Geochem. J. 28, 263-287. doi:10.2343/geochemj.28.263

Saishu, H., Okamoto, A., and Tsuchiya, N. (2014). The Significance of Silica Precipitation on the Formation of the Permeable-Impermeable Boundary within Earth's Crust. Terra Nova 26, 253-259. doi:10.1111/ter.12093

Shinohara, H., and Fujimoto, K. (1994). Experimental Study in the System AlbiteAndalusite-Quartz-NaCl-HCl-H2o at $600^{\circ} \mathrm{C}$ and 400 to 2000 Bars. Geochimica et Cosmochimica Acta 58, 4857-4866. doi:10.1016/0016-7037(94)90216-X

Shinohara, H., Ohba, T., Kazahaya, K., and Takahashi, H. (2008). Origin of Volcanic Gases Discharging from a Cooling Lava Dome of Unzen Volcano, Japan. J. Volcanology Geothermal Res. 175, 133-140. doi:10.1016/ j.jvolgeores.2008.03.024

Stix, J., and de Moor, J. M. (2018). Understanding and Forecasting Phreatic Eruptions Driven by Magmatic Degassing. Earth Planets Space 70, 83. doi:10.1186/s40623-018-0855-z

Takahashi, K., and Fujii, I. (2014). Long-term thermal Activity Revealed by Magnetic Measurements at Kusatsu-Shirane Volcano, Japan. J. Volcanology Geothermal Res. 285, 180-194. doi:10.1016/j.jvolgeores.2014.08.014

Takahashi, M., Kawamata, H., Yasui, M., and Kanamaru, T. (2010). Whole Rock Major Element Chemistry for Eruptive Products of Kusatsu-Shirane Volcano, central Japan: Summary of 306 Analytical Data. Proc. Inst. Nat. Sci. Nihon Univ. $45,205-254$. (in Japanese).

Takano, B., Kuno, A., Ohsawa, S., and Kawakami, H. (2008). Aqueous Sulfur Speciation Possibly Linked to Sublimnic Volcanic Gas-Water Interaction during a Quiescent Period at Yugama Crater lake, Kusatsu-Shirane Volcano, Central Japan. J. Volcanology Geothermal Res. 178, 145-168. doi:10.1016/ j.jvolgeores.2008.06.038

Takano, B., and Watanuki, K. (1990). Monitoring of Volcanic Eruptions at Yugama Crater lake by Aqueous Sulfur Oxyanions. J. Volcanology Geothermal Res. 40, 71-87. doi:10.1016/0377-0273(90)90107-Q

Taran, Y. A., Pokrovsky, B. G., and Doubik, M. (1989). Isotopic Composition and Origin of Water from Andesitic Magmas. Dokl. Acad. Sci. USSR 304, 440-443.

Terada, A., Kanda, W., Ogawa, Y., Yamada, T., Yamamoto, M., Ohkura, T., et al. (2021). The 2018 Phreatic Eruption at Mt. Motoshirane of Kusatsu-Shirane Volcano, Japan: Eruption and Intrusion of Hydrothermal Fluid Observed by a Borehole Tiltmeter Network. Earth Planets Space 73, 157. doi:10.1186/s40623021-01475-4
Terada, A. (2018). Kusatsu-Shirane Volcano as a Site of Phreatic Eruptions. Jour. Geol. Soc. Jpn. 124, 251-270. doi:10.5575/geosoc.2017.0060

Terada, A., Morita, Y., Hashimoto, T., Mori, T., Ohba, T., Yaguchi, M., et al. (2018). Water Sampling Using a Drone at Yugama Crater lake, KusatsuShirane Volcano, Japan. Earth Planets Space 70, 64. doi:10.1186/s40623-0180835-3

Tokyo Institute of Technology and Sophia University (1983). The Activity of Kusatsu-Shirane Volcano in 1982 and Geochemical Study (II). Rep. Coord. Commit. Pred. Volc. Eruption 28, 7-18. (in Japanese).

Tokyo Institute of Technology, Sophia University, and Saitama University (1983). The Activity of Kusatsu-Shirane Volcano in October 1982 and Geochemical Study (I). Rep. Coord. Commit. Pred. Volc. Eruption 26, 8-19. (in Japanese).

Tseng, K. H., Ogawa, Y., Tank, S. B., Ujihara, N., Honkura, Y., Terada, A., et al. (2020). Anatomy of Active Volcanic Edifice at the Kusatsu-Shirane Volcano, Japan, by Magnetotellurics: Hydrothermal Implications for Volcanic Unrests. Earth Planets Space 72, 161. doi:10.1186/s40623-020-01283-2

Ueki, K., and Terada, A. (2012). Field Excursion Guide to Kusatsu-Shirane Volcano. Bull. Volcanol. Soc. Jpn. 57, 235-251. (in Japanese). doi:10.18940/ kazan.57.4_235

Uto, K., Hayakawa, Y., Aramaki, S., and Ossaka, J. (1983). Geological Map of Kusatsu-Shirane Volcano. Ibaraki: Geological Survey of Japan.

Varekamp, J. C. (2015). "The Chemical Composition and Evolution of Volcanic Lakes," in Volcanic Lakes, Advances In Volcanology. Editors D. Rouwet, B. Christenson, F. Tassi, and J. Vandemeulebrouck (Heidelberg: Springer), 93-123. doi:10.1007/978-3-642-36833-2_4

Volcanic Fluid Research Center, Tokyo Institute of Technology (2004). Recent Activity of Kusatsu-Shirane Volcano-Water Jet from Crater lake Yugama on 17, May 2004. Rep. Coord. Commit. Pred. Volc. Eruption 88, 49-54. (in Japanese).

Yaguchi, M., Muramatsu, Y., Chiba, H., Okumura, F., and Ohba, T. (2016) The Origin and Hydrochemistry of Deep Well Waters from the Northern Foot of Mt. Fuji, central Japan. Geochem. J. 50, 227-239. doi:10.2343/ geochemj.2.0409

Yamaoka, K., Geshi, N., Hashimoto, T., Ingebritsen, S. E., and Oikawa, T. (2016). Special Issue "The Phreatic Eruption of Mt. Ontake Volcano in 2014". Earth Planets Space 68, 175. doi:10.1186/s40623-016-0548-4

Conflict of Interest: The authors declare that the research was conducted in the absence of any commercial or financial relationships that could be construed as a potential conflict of interest.

Publisher's Note: All claims expressed in this article are solely those of the authors and do not necessarily represent those of their affiliated organizations, or those of the publisher, the editors and the reviewers. Any product that may be evaluated in this article, or claim that may be made by its manufacturer, is not guaranteed or endorsed by the publisher.

Copyright (c) 2021 Yaguchi, Ohba and Terada. This is an open-access article distributed under the terms of the Creative Commons Attribution License (CC $B Y)$. The use, distribution or reproduction in other forums is permitted, provided the original author(s) and the copyright owner(s) are credited and that the original publication in this journal is cited, in accordance with accepted academic practice. No use, distribution or reproduction is permitted which does not comply with these terms. 